\title{
Review
}

Marcin Poreba*

\section{Recent advances in the development of legumain- selective chemical probes and peptide prodrugs}

https://doi.org/10.1515/hsz-2019-0135

Received January 29, 2019; accepted April 18, 2019; previously published online May 22, 2019

Abstract: Legumain, which is also known as vacuolar processing enzyme (VPE) or asparaginyl endopeptidase (AEP), is a cysteine protease that was first discovered and characterized in the leguminous seeds of the moth bean in the early 1990s. Later, this enzyme was also detected in higher organisms, including eukaryotes. This $\mathrm{pH}$-dependent protease displays the highest activity in acidic endolysosomal compartments; however, legumain also displays nuclear, cytosolic and extracellular activity when stabilized by other proteins or intramolecular complexes. Based on the results from over 25 years of research, this protease is involved in multiple cellular events, including protein degradation and antigen presentation. Moreover, when dysregulated, this protease contributes to the progression of several diseases, with cancer being the well-studied example. Research on legumain biology was undoubtedly facilitated by the use of small molecule chemical tools. Therefore, in this review, I present the historical perspectives and most current strategies for the development of small molecule substrates, inhibitors and activity-based probes for legumain. These tools are of paramount importance in elucidating the roles of legumain in multiple biological processes. Finally, as this enzyme appears to be a promising molecular target for anticancer therapies, the development of legumain-activated prodrugs is also described.

Keywords: activity-based probes; asparaginyl endopeptidase; inhibitors; legumain; prodrugs; substrates.

\section{Introduction}

Legumain is a lysosomal protease that was originally described in trematode Schistosoma mansoni (Ruppel

\footnotetext{
*Corresponding author: Marcin Poreba, Department of Bioorganic Chemistry, Wroclaw University of Science and Technology, Wyb. Wyspianskiego 27, 50-370, Wroclaw, Poland, e-mail:marcin.poreba@pwr.edu.pl
}

et al., 1987; Klinkert et al., 1989) as well as in leguminous seeds (Kembhavi et al., 1993) and later discovered and characterized in higher eukaryotes (Tanaka et al., 1996; Chen et al., 1997). This protease, along with caspases, clostripains, separases and gingipains, belong to the clan CD, and family C13 of proteolytic enzymes (Chen et al., 1998; Dall and Brandstetter, 2016; Rawlings et al., 2018). The unique characteristic of this clan is the narrow substrate specificity of its members for the P1 position (Schomburg et al., 2004), i.e. caspases cleave substrates after aspartic acids (Poreba et al., 2015), gingipains and clostripains prefer Arg and Lys (Cole et al., 1971; Ally et al., 2003), separases exclusively recognize Arg (Winter et al., 2015), and legumain hydrolyzes the peptide bond after Asn and, to a lesser extent, after Asp residues (Vidmar et al., 2017). Moreover, CD proteases also share the conserved His-Gly-spacer-Ala-Cys catalytic motif, which suggests an evolutionary relationship between these enzymes (Chen et al., 1998; Dall and Brandstetter, 2016). All members of the CD clan play key roles in multiple cellular pathways, including cell death, the cell cycle, inflammation and antigen presentation (Rawlings et al., 2018).

Given its high preferences for processing substrates after Asn, legumain was named asparaginyl endopeptidase (AEP), and the names legumain and AEP are currently used interchangeably (Dall and Brandstetter, 2016). The biological role of legumain is well studied, and this protease is generally accepted to participate in a number of critical cellular pathways and biological processes (Brix et al., 2015; Zhen et al., 2015; Dall and Brandstetter, 2016). For instance, the proteolytic activity of legumain is important for protein processing and antigen presentation to the MHC II complex (Manoury et al., 1998; Matthews et al., 2010). Moreover, by hydrolyzing cysteine cathepsins, which also participate in antigen processing, legumain is a downstream modulator of the immune response (Maehr et al., 2005). The proteolytic activity of legumain is controlled at different levels, such as proenzyme expression, pH-dependent activity or complex formation with endogenous inhibitors (Briggs et al., 2010; Dall and Brandstetter, 2012, 2013; Smith et al., 2012). The deregulation of legumain activity is associated with multiple 
pathophysiological events, with cancer being the most prominent example (Mai et al., 2017). In 2003, researchers first reported legumain overexpression in multiple types of solid tumors, including breast, colon, lung, prostate and ovarian cancer (Liu et al., 2003). Elevated legumain activity was subsequently correlated with enhanced cancer growth and metastasis, and positively correlated with a poor prognosis for patients (Liu et al., 2003; Guo et al., 2013; Li et al., 2013). Moreover, legumain was also detected in tumor-adjacent cells, such as tumor-associated macrophages (TAMs), which are important regulators of cancer cells proliferation and invasiveness (Liu et al., 2003; Lin et al., 2013). The tumor microenvironment is generally characterized by a low $\mathrm{pH}$, allowing legumain to be catalytically active both intra- and extracellularly (Dall and Brandstetter, 2013). Finally, cancer-related legumain activity is also modulated by natural inhibitors (AlvarezFernandez et al., 1999; Schomburg et al., 2004). The loss of cystatin $\mathrm{E} / \mathrm{M}$, a potent legumain inhibitor, the results in the increased tumor growth (Briggs et al., 2010). In addition to cancer, legumain is also upregulated in Alzheimer's disease (Basurto-Islas et al., 2013; Zhang et al., 2014) and multiple sclerosis lesions (Manoury et al., 2002). The association of legumain with various pathophysiological events stresses the need for the development of chemical tools that selectively and accurately detect this protease (Mai et al., 2017).

Legumain is synthesized as a catalytically inactive zymogen $(56 \mathrm{kDa})$ that undergoes autocatalytic processing under acidic conditions, resulting in the formation of two intermediates $(47 \mathrm{kDa}$ and $48 \mathrm{kDa}$ ) that are further processed by other cysteine proteases into the mature, active form (36 kDa) (Figure 1A) (Dall and Brandstetter, 2016). The activity of mature legumain is controlled by endogenous type 2 cystatins, such as cystatin E/M,
C and F (Alvarez-Fernandez et al., 1999; Cheng et al., 2006; Wallin et al., 2017; Dall et al., 2018). Although the proenzyme is stable at neutral $\mathrm{pH}$, catalytically active uncomplexed legumain is stable only at acidic $\mathrm{pH}$ and undergoes irreversible destabilization at higher $\mathrm{pH}$ values (Dall and Brandstetter, 2013) (Figure 1B). For this reason, legumain is mainly located in the acidic compartments of the endolysosomal system. However, this protease is also active extracellularly, but only when bound to integrins, which stabilize its tertiary structure or when the extracellular microenvironment is slightly acidic (i.e. cancer disease) (Liu et al., 2003, 2012; Dall and Brandstetter, 2013; Kato et al., 2013). In addition, legumain can also display nuclear and cytosolic localization. It has been demonstrated that under pathological conditions (such as an Alzheimer's disease, $\mathrm{AD}$ ) legumain may be released from the neuronal lysosomes to the cytosol, and then enter the nucleus (Basurto-Islas et al., 2013; Zhang et al., 2014; Dall and Brandstetter, 2016). Importantly, the translocated legumain is highly active in the cytosol, while its activity in the nuclear fraction is negligible (Basurto-Islas et al., 2013). Cancer is another disease where legumain is found to be active outside the lysosomes. For instance, Haugen and co-workers demonstrated that in colorectal cancer significant activity of legumain can be found in lysosomal and nuclear fractions, and to a lesser extent in a cytosol (Haugen et al., 2013, 2015). The possible explanation of legumain retaining its catalytic activity in the cytosol and nucleus, is that in both AD and cancer these cell compartments tend to be slightly acidified. However, even at neutral $\mathrm{pH}$ legumain can be still active when stabilized by other proteins or intramolecular complexes (Dall and Brandstetter, 2016).

As legumain shares approximately $15 \%$ overall sequence homology with caspase-1, it is very often
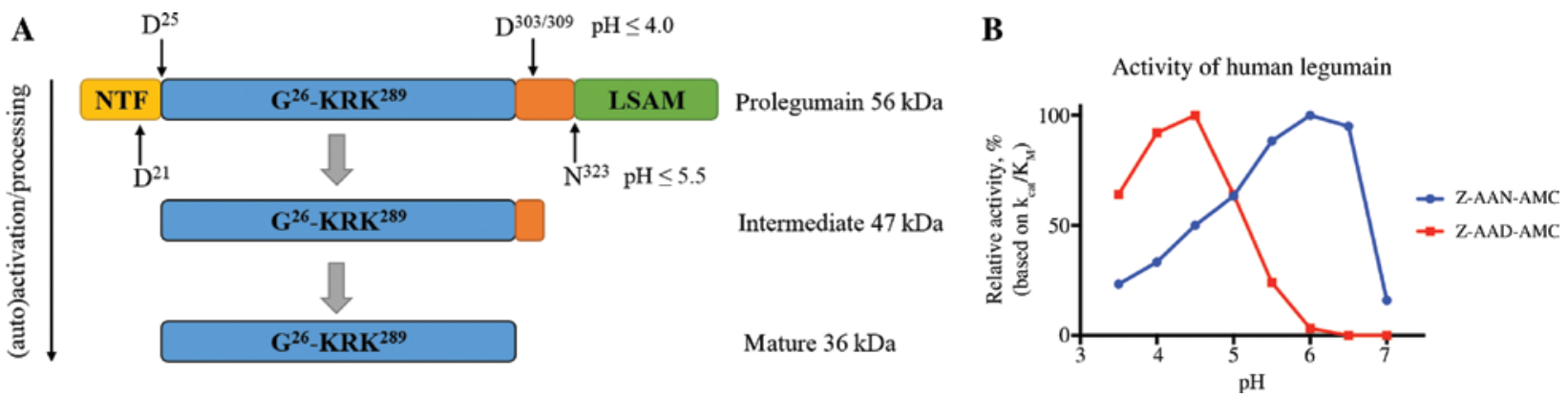

Figure 1: Legumain activation and catalytic activity.

(A) The mechanism of legumain (auto)-processing and activation. Legumain is synthesized as $56 \mathrm{kDa}$ proenzyme and undergoes sequential processing to $36 \mathrm{kDa}$ catalytically active mature form. Cartoon adapted from Dall and Brandstetter (2016). (B) Legumain displays pH-dependent activity with the maximum at $\sim$ pH 6.0 (Z-AAN-AMC substrate, blue). At lower pH legumain can also hydrolyzes substrates with ASP at the P1 position (Z-AAD-AMC, red). Adapted from Vidmar et al. (2017). 
considered a caspase-like enzyme, but biochemically, legumain is more closely related to cysteine cathepsins (Dall and Brandstetter, 2016). This feature is reflected in the same endolysosomal localization, $\mathrm{pH}$-dependent activation and catalytic activity, and the fact that legumain, similar to cathepsins, is inhibited by cystatins. However, the most striking difference between legumain and cathepsins is that legumain is not sensitive to E-64, a potent and broad spectrum cathepsin inhibitor (Chen et al., 1997; Schomburg et al., 2004). This biochemical characteristic makes legumain a very interesting enzyme in the context of the development of potent and selective substrates and inhibitors that distinguish between legumain and caspases or cathepsins. In this review, I present the historical perspectives of prototypic legumain substrates and inhibitors, and describe the recent progress in the pursuit of highly selective legumain tools for the detection of this protease in complex biological systems, ranging from cell cultures to whole animal live imaging.

In addition to the proteolytic activity, legumain also displays the ligase activity (Dall et al., 2015; Jackson et al., 2018; Zauner et al., 2018), which was demonstrated for the first time in 1993 by Ishii (Ishii, 1993). However, in contrast to the hydrolytic activity which is much more pronounced at acidic pH (Vidmar et al., 2017), the ligase activity dominates at the neutral pH (Dall et al., 2015). The key structural feature for legumain ligase activity is Asp147 residue located in the active site, which during the autocatalytic activation is converted into an energy-rich aspartimide Asu147 (also called Suc147, succinimide) (Zhao et al., 2014; Dall et al., 2015). This posttranslational modification is crucial for the ligase activity, as it was shown that the mutation of Asp147 into Ser147 or Gly147 completely abolishes the legumain ability to re-ligate (resynthesize) cystatin E protein (Dall et al., 2015). The generally accepted mechanism of the ligation performed by legumain involves four steps and has been recently reviewed by Dall and
Brandstetter (2016). In the first step legumain cleaves its substrate to generate free C-terminal fragment $(-\mathrm{COOH})$, which can further attack energy-rich Asu147 residue resulting in the formation of carboxylate anhydride. Next, this anhydride (electrophile) is attacked by the N-terminal free peptide (nucleophile) which leads to the formation of a peptide bond and regeneration of an enzyme. However, the presented mechanisms is not the only option, as legumain may also ligate substrates through a sortase-like mechanism, which involves the formation of a classical thiohemiketal complex between P1 Asp and catalytic Cys189, followed by the release of a cleavage product and the nucleophilic substitution reaction with another free C-terminal substrate (Bernath-Levin et al., 2015) (Figure 2). The ligase substrate specificity of legumain is determined by the preferences of the $\mathrm{S} 1$ pocket, that can accommodate only Asn and to a lesser extent Asp. Once the substrate is cleaved by legumain, a new C-terminal fragment with P1 Asn or Asp is generated and serves as a substrate for the ligation reaction. As the ligase activity of legumain is very well-studied in plants, multiple such substrates have been already described (Dall and Brandstetter, 2016). One of the most prominent examples is the PawS1 protein, a precursor of an SFTI-1 (sunflower trypsin inhibitor 1). This protein is first hydrolyzed by legumain at Asn35 and Asp49 and then the 14-amino acids fragment is head-totail ligated (Gly36 to Asp49) to form a cyclic peptide known as SFTI (Figure 2) (Mylne et al., 2011). Another examples of substrates for the ligation reaction performed by plant legumain are prolegumain itself (Kuroyanagi et al., 2002), proconcanavalin A (Sheldon et al., 1996), kalatatype cyclic peptides (Gillon et al., 2008) or cyclic knottins (Mylne et al., 2012). In humans an important example of legumain protease-ligase activity is its binding to cystatin E, a potent cysteine proteases inhibitor. Dall and coworkers demonstrated that the incubation of legumain with cystatin $\mathrm{E}$ at $\mathrm{pH} 4.0$ leads to the rapid hydrolysis of

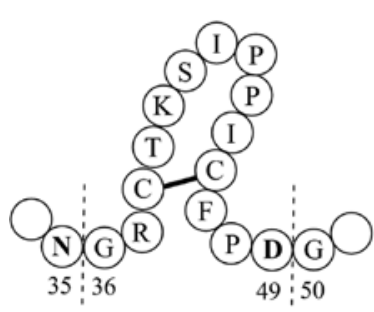

PawS1 protein

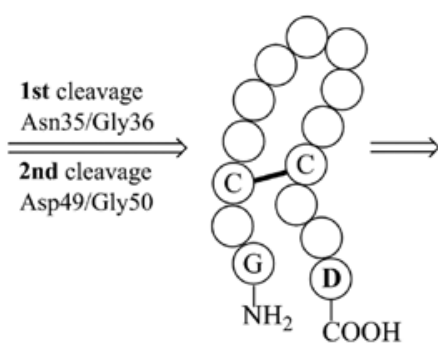

Cleaved peptie

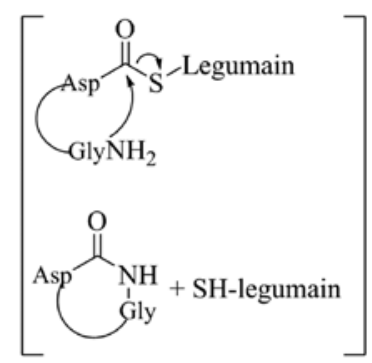

Ligation

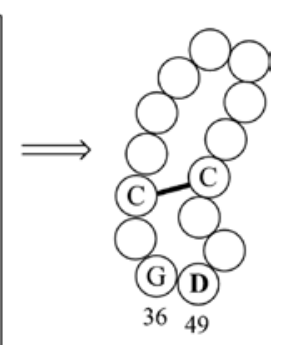

Cyclic SFTI-1

Figure 2: Schematic representation of a PawS1 protein hydrolysis by legumain followed by peptide ligation to form cyclic sunflower trypsin inhibitor 1 (SFTI-1).

In this case the ligation (transpeptidation reaction) is performed by legumain via sortase-like mechanisms, which involves a direct nucleophilic attack on a peptide bond and coupling to the N-terminal fragment of a new peptide. Adapted from Mylne et al. (2011). 
Asn39-Ser40 peptide bond, which can be further re-ligated by the enzyme at the $\mathrm{pH}$ 6.5. Moreover, authors found that this re-ligation is independent of catalytic Cys189, as even non-proteolytically active legumain can still perform ligation reaction (Dall et al., 2015). Therefore, by describing the legumain protease-ligase activity, this study provided a detailed mechanistic insight on how the cystatin $\mathrm{E}$ binds and inhibits AEP.

\section{Legumain substrates and substrate- based probes}

The most unique feature of legumain is its narrow specificity to hydrolyze substrates after Asn residues (P1 position) (Rawlings et al., 2018). The first synthetic substrates for legumain (isolated from the moth bean) were developed by Kembhavi et al. (1993). Legumain preferentially recognizes Z-Ala-Ala-Asn-AMC $\left(k_{\text {cat }} / K_{M}=10400 \mathrm{M}^{-1} \mathrm{~s}^{-1}\right)$ and, to a lesser extent, Z-Ala-Pro-Asn-AMC (Figure 3). This discovery was reported over 25 years ago, and these substrates are currently the gold standards in countless legumain-based kinetic assays. In addition to substrate development, the authors also revealed that legumain is not sensitive to the E-64 inhibitor and displays only negligible reactivity towards cystatin or leupeptin. Thus, the authors speculated that legumain must be an atypical cysteine protease. In the same year, legumain (called here: vacuolar processing enzyme, VPE) from the $S$. mansoni parasite was also characterized (Hara-Nishimura et al., 1993). The primary specificity of legumain for Asn at the P1 position was confirmed by the observation that this protease cleaved the SESENGLEET decapeptide between the Asn and Gly residues. Shortly after this report, the first cloning, isolation and biochemical characterization of mammalian legumain was described by Chen and coworkers (Chen et al., 1997). Using the prototypic Z-Ala-Ala-Asn-AMC substrate $\left(k_{\text {cat }} / K_{M}=920000 \mathrm{M}^{-1} \mathrm{~S}^{-1}\right)$, the authors showed that the maximal activity of pig legumain occurs at $\mathrm{pH}$ 5.8, and the enzyme is irreversibly denatured at a $\mathrm{pH}$ greater than 7.0. The high specificity of legumain for the Z-Ala-Ala-AsnAMC substrate allowed researchers to test its enzymatic activity in extracts of various tissues from rats, pigs and rabbits. Legumain displayed the highest activity in kidney and spleen, whereas the activity in other tissues (liver, lung and the intestine) was negligible.

The first report on human legumain kinetics was provided by Halfon et al. (1998). The authors expressed active legumain in eukaryotic cell cultures and incubated it with the Z-Ala-Ala-Asn-AMC $\left(k_{\text {cat }} / K_{M}=200000 \mathrm{M}^{-1} \mathrm{~S}^{-1}\right)$ and Z-Gly-Ala-Gln-AMC (no hydrolysis) substrates, confirming the substantial preference of this protease for Asn at P1. Moreover, legumain was also shown to slowly hydrolyze the succinyl-YVAD-AMC substrate at $\mathrm{pH}$ 3. The ability of legumain to cleave after Asp residues was further shown to be essential for enzyme autoactivation under acidic conditions. The optimal Z-Ala-Ala-Asn tripeptide scaffold was later used by Johansen et al. to develop the first colorimetric assay for the detection of legumain activity (Figure 3) (Johansen et al., 1999). The use of a 4-methoxy-2-napthylamine (NH-NapOMe) group not only allowed the authors to measure legumain kinetics $\left(k_{\text {cat }} / K_{M}=250000 \mathrm{M}^{-1} \mathrm{~s}^{-1}\right)$ and screen potential inhibitors but also to visualize active legumain after electrophoresis in a polyacrylamide gel. The authors also speculated that the proposed substrate could be used for the histochemical staining of legumain activity in tissue sections, as the NH-NapOMe leaving group from the substrate forms an insoluble complex with 5-nitrosalicylaldehyde. Indeed, in later studies, this approach was utilized to detect active legumain in the skin of cystatin E/M-deficient mice (Zeeuwen et al., 2004) and in colorectal
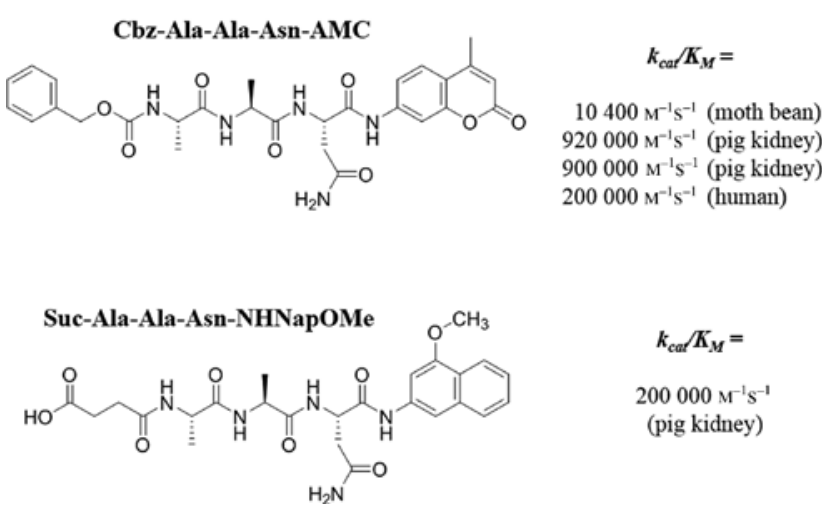

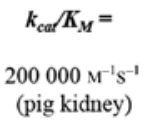

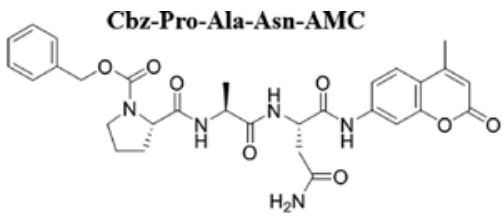

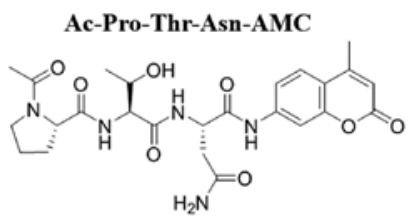

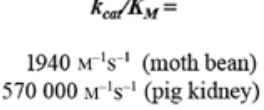

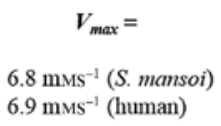

Figure 3: Structures of classical fluorogenic substrates to study legumain activity. 
cancer xenografts (Haugen et al., 2013). Although substrates with the Ala-Ala-Asn scaffold were typically used to detect legumain activity at that time, little was known about the specificity of this enzyme for amino acids in the P3-P2 positions. One of the first reports addressing this issue was published by Dando et al. (1999). By utilizing a series of Z-X-Ala-Asn-AMC peptides, the authors found that pig legumain prefers small (Ala, Val, and Pro) and bulky and hydrophobic $[\operatorname{Tyr}(t \mathrm{Bu})$, Phe, and Tyr] amino acids in the P2 position (Figure 4A). Moreover, no substrate cleavage was detected for substrates containing mono- or di-Nmethylasparagine residues in the $\mathrm{P} 1$ position, confirming the high degree of specificity for Asn in the S1 pocket of legumain. Shortly thereafter, similar studies were conducted by Rotari et al. (2001). Using Z-Xaa-Ala-Asn-AMC substrates, the authors reported similar $\mathrm{pH}$-dependent activity profiles for kidney bean and pig legumain, with the maximal activity at $\mathrm{pH}$, but revealed distinct specificity profiles in the P3 position. Kidney bean legumain preferred $\operatorname{Tyr}(t \mathrm{Bu})$, Val, Phe and Tyr amino acids over Ala, whereas pig legumain favored Ala over bulky, hydrophobic amino acids. These $\mathrm{P} 3$ discrepancies reflect the general differences in the hydrolysis of natural substrates, which in turn indicates that pig and kidney bean legumain variants have distinct physiological roles. Another variant of the legumain protease was detected in the parasitic blood fluke S. mansoni (Dalton and Brindley, 1998). This enzyme was reported to serve as good diagnostic marker for schistosomiasis, thus the identification of its substrate preferences will help researchers develop substrate-based diagnostic tools or therapeutic inhibitors. In 2002, Mathieu and colleagues applied the PS-SCL approach (Positional Scanning Synthetic Combinatorial Library) to screen the substrate specificity of parasitic (S. mansoni) and mammalian (human) legumain at the P3-P2 positions (Figure 4B)
(Mathieu et al., 2002). The parasitic protease has strong preferences for Thr/Ala/Ser/Pro amino acids in the P3 position and Ala/Ser/Thr/Pro in the P2 position. The specificity of human legumain was similar, with Pro/Thr being the best recognized amino acids in the $\mathrm{P} 3$ position, and Thr in P2 (Figure 3). Although, slight differences in the recognition of individual amino acids were observed, the overall specificity of these enzymes was highly homologous, as reflected by the kinetic analysis of individual substrates. For example, the optimal substrate for human legumain, Ac-PTN-AMC, was hydrolyzed at a similar rate by both enzymes, whereas the optimal substrate for schistosomal legumain, Ac-TAN-AMC, was hydrolyzed at an even faster rate by the human orthologue than by the parasitic protease. In the same year, the specificity of legumain towards the $\mathrm{P}^{\prime}$ position was also investigated. Schwarz and coworkers utilized a 20-member panel of label-free peptides with the general formula of Z-AEN/XAEK- $\mathrm{NH}_{2}$ (' $/$ ' is the cleavage site) to show that pig legumain recognizes almost all amino acids equally, with a lower tolerance only observed for Pro (Figure 4C) (Schwarz et al., 2002). Thus, the P1' position has almost no impact on legumain-mediated substrate turnover.

A screen of legumain specificity in the prime region is useful for predicting its natural substrates (Dando et al., 1999; Schwarz et al., 2002). However, the use of label-free peptides does not provide a direct readout, making the analysis more time- and labor-consuming. An alternative approach is to use internally quenched fluorescent (IQF) substrates, where the peptide sequence is flanked by a donor (fluorophore) and an acceptor (quencher). Upon the hydrolysis of a peptide bond, the fluorophore and quencher are separated, the fluorophore is no longer quenched, and enhanced fluorescence is generated. In 2016, Poreba et al. described the synthesis and biochemical evaluation
A
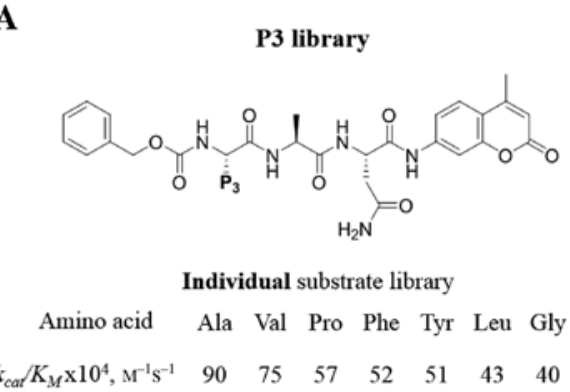

B

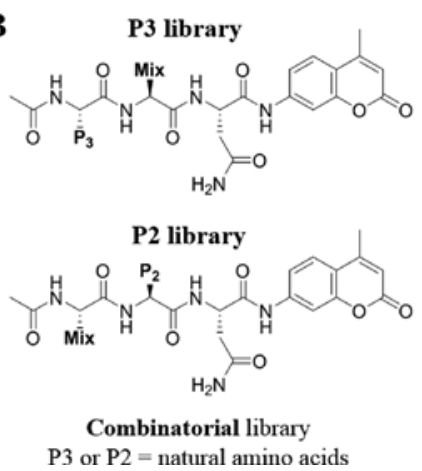

C

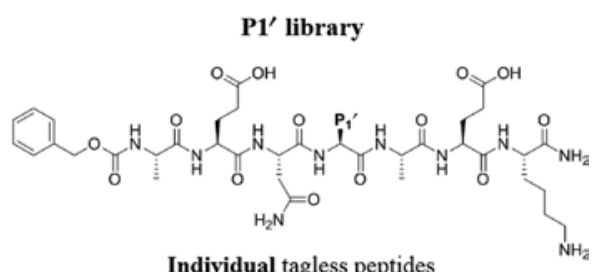
$\mathrm{Pl}^{\prime}$ - natural amino acids

Figure 4: Dissecting the legumain specificity with various substrate libraries.

(A) A library of individual AMC-tagged substrates to study P3 preferences. (B) Two combinatorial fluorogenic substrate libraries to study P3 and P2 preferences. (C) A library of individual tagless peptides to dissect legumain specificity at the S1' active site pocket. 
of two IQF substrates with MCA or ACC fluorophores and the Lys(Dnp) acceptor (Poreba et al., 2017b). Both these substrates contained the donor-GTPN/KVK(Dnp)R-NH sequence and were efficiently hydrolyzed by human legumain $\left(k_{\text {cat }} / K_{M}=149000 \mathrm{M}^{-1} \mathrm{~s}^{-1}\right.$ for the ACC substrate and $139000 \mathrm{M}^{-1} \mathrm{~S}^{-1}$ for the MCA substrate) (Figure 5A). This study highlights the usefulness of IQF substrates in the profiling of legumain specificity for the prime positions, a region which is inaccessible using traditional peptide$\mathrm{AMC} / \mathrm{ACC}$ substrates. Internally quenched fluorescent substrates have also been developed by including two fluorophores in one substrate molecule, where one of fluorophores serves as a quencher. This approach was realized by Chen and coworkers, who developed a nearinfrared (NIR) fluorescent legumain probe by flanking a legumain-selective peptide (Ala-Ala-Asn/Leu (where '/, represents the cleavage site) with the CyTE777 (donor) and CyTE807 (acceptor) fluorochromes (Figure 5B) (Chen et al., 2014). In close proximity, the fluorescence from CyTE777 is quenched by the CyTE807 fluorochrome, but upon legumain cleavage, this energy transfer disappears and increased fluorescence from CyTE777 is observed. The utility of this probe for legumain detection was proven in 3T3 cells and the optical imaging of mice bearing CT26 tumor xenografts. The great advantage of this probe is its NIR characteristic (excitation: $745 \mathrm{~nm}$, emission: $820 \mathrm{nM}$ ); thus, the signals from these fluorophores penetrate deep in tissues, allowing in vivo imaging. In addition to this IQF probe, the authors developed an MRI contrast agent with a peptide cleaved by legumain and a gadolinium ion $\left(\mathrm{Gd}^{3+}\right)$ complexed by the NBCB chelator (Chen et al., 2014). In principle, legumain-mediated cleavage of this substrate-like probe allows the Gd-containing fragment to bind to human serum albumin (HSA) and form macromolecules, which in turn results in an increased magnetic resonance imaging (MRI) signal. This probe was successfully applied to detect legumain activity in mice bearing CT26 xenografts; thus, the authors speculate that this newly designed Gd-probe should also be applied to the in vivo imaging of other proteases in various tumors. Shortly thereafter, another MRI-suitable probe for legumain detection was developed. Yuan and coworkers synthesized a legumain-sensitive peptidic probe labeled with the ${ }^{19} \mathrm{~F}$ contrast agent and equipped with a 2-cyanobenzothiazole (CBT) group, facilitating glutathione-controlled self-assembly (Yuan et al., 2015). Once the probe enters cells, it self-assembles into nanoparticles (NPs) upon glutathione reduction, followed by legumain-mediated disassembly, which in turn activates the ${ }^{19} \mathrm{~F}$ NMR/MRI signal. This probe was shown to capture legumain activity in HEK293T cells and a tumor-bearing zebrafish model, thus appearing to serve as another substrate-based system for legumain-selective imaging. The same hybrid nanoprobe platform was later used by Zhao and coworkers (Zhao et al., 2018). However, instead of using the ${ }^{19} \mathrm{~F}$ isotope, the authors decorated the probe with the Cy5.5 fluorophore, which upon glutathione-mediated self-assembly becomes nonfluorescent ('off') through an aggregation-caused
A

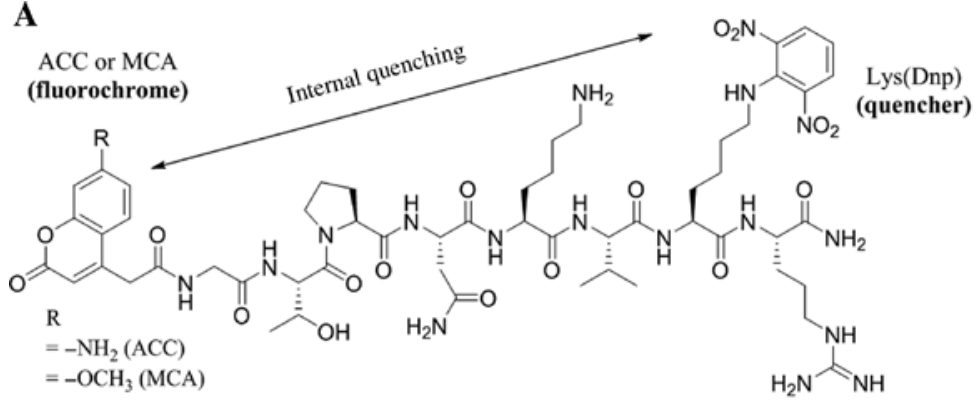

B

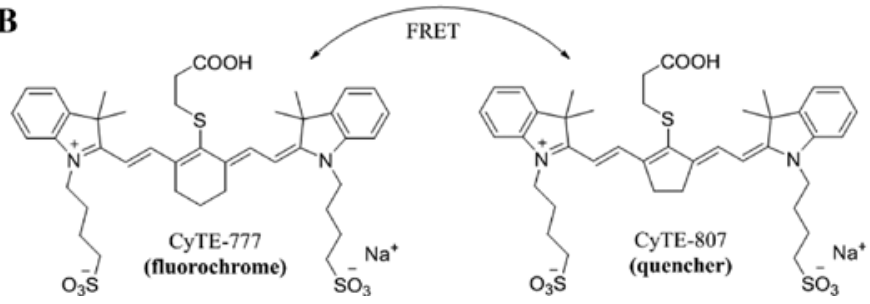

C
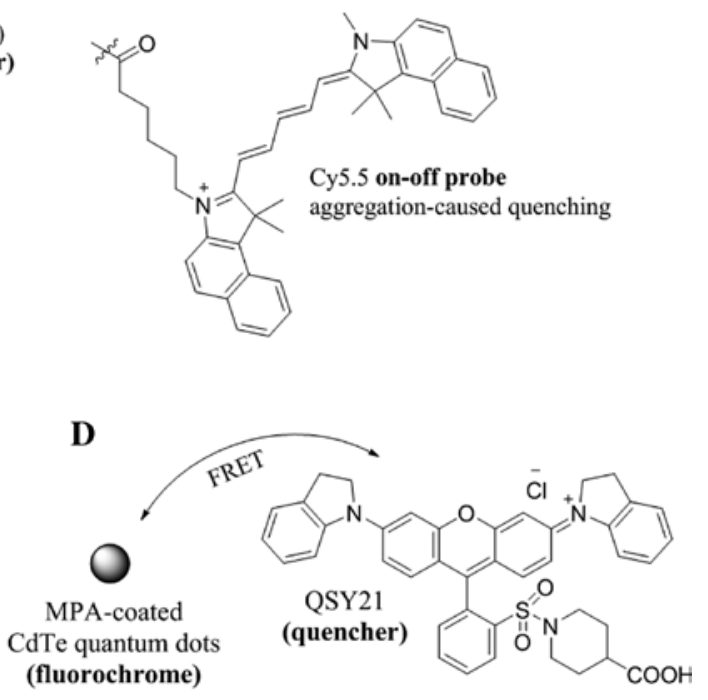

Figure 5: Selected examples of fluorophores and quenchers used in the design of internally-quenched fluorescent substrates (IQFS) and fret substrates to study legumain activity. 
quenching (ACQ) effect (Figure 5C). However, in the presence of legumain, the peptide is released from the NPs, resulting in the recovery of the fluorescence signal ('on'). This probe was shown to be useful for detecting legumain in cultured cancer cells and tumor-bearing mice. Another report describing the development of a legumainactivated nanoprobe was presented by Wang and coworkers (Wang et al., 2014). In this construct, fluorescent quantum dots (QD) modified with low-molecular weight heparin (LH) were coupled with a fluorescence quencher (QSY21) through a legumain-labile (PTN/G) peptide to obtain a Förster resonance energy transfer (FRET) probe (Figure 5D). The reported probe was equipped with a protamine peptide (VSRRRRRRGGRRRR) to increase cellular uptake. Although this probe showed good sensitivity towards legumain in a test tube, it also exhibited some off-target cross-reactivity with trypsin. Importantly, the authors confirmed that this FRET substrate was useful for detecting legumain activity in cultured cells and in tumorbearing mice in vivo. This QD/QSY system was particularly focused on detecting legumain; however, the authors concluded that this QD nanoprobe platform can also be applied to detect other tumor-associated proteases.

The profiling of legumain substrate specificity is not limited to the use of synthetic fluorescence substrates. According to multiple reports, the hydrolysis of peptides and proteins by recombinant or isolated legumain can also provide more information about the catalytic preferences of legumain and therefore provide insights into its putative biological roles. However, these methods suffer from the inability to create a complete picture of the legumain substrate specificity. In 2017, Vidmar and coworkers developed a gel-based label-free proteomic approach (called DIPPS) to discover the broad substrate preferences of proteases (Vidmar et al., 2017). Using DIPPS, the authors did not observe a strong preference for legumain in the active site pockets, except for the P1 position, where only Asn and Asp are recognized. Importantly, this legumain $\mathrm{P} 1$ specificity is $\mathrm{pH}$-dependent, as legumain displays the highest activity towards substrates with Asn in $\mathrm{pH}$ values ranging from 5.5 to 6.5 , whereas P1-Asp substrates are preferentially hydrolyzed under more acidic conditions $(\mathrm{pH}$ ranging from 3.5 to 5.0). This shift in the primary specificity of legumain can be explained by the observation that Asp is protonated at low $\mathrm{pH}$ and thus resembles Asn. Therefore, protonated Asp fits in the legumain S1 pocket (Dall and Brandstetter, 2013, 2016). The ability of legumain to cleave substrates after Asp residues suggests that the specificity of this protease may overlap with caspases, which are known to have almost absolute preferences for this amino acid in the P1 position (Thornberry et al., 1997). This common characteristic makes the development of legumain-selective substrates with Asp in the P1 position very challenging. In 2016, Poreba and coworkers overcame this problem by employing natural and a large panel of unnatural amino acids to screen legumain preferences in the P4-P2 positions (Figure 6) (Poreba et al., 2016). The application of a variety of unnatural amino acids in the peptide library called HyCoSuL (Hybrid Combinatorial Substrate Library) facilitated a detailed exploration of the
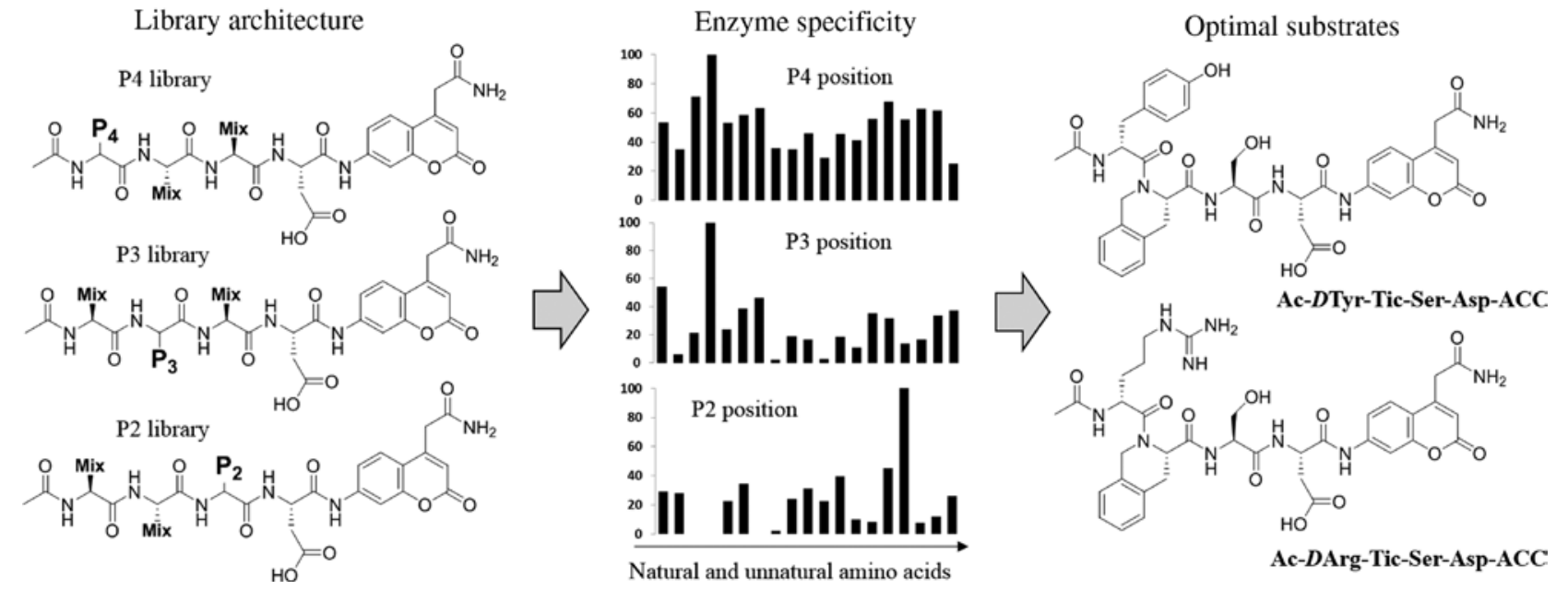

Figure 6: Development of legumain selective substrates through HyCoSuL profiling. $\mathrm{HyCoSuL}$ is a P4-P2 combinatorial fluorogenic substrate library that contain natural and a wide range of unnatural amino acids, making possible to deeply explore the chemical space in the protease active site. 
chemical space in the protease active site and dramatically increased the chances of identifying amino acids that distinguish between legumain and caspases (Poreba et al., 2017a). Based on the HyCoSuL screening data, legumain displays high preferences for Thr in the P2 position and for the unnatural amino acid Cys(Bzl) (and its derivatives) in the P3 position. Interestingly, legumain has no preferences in the $\mathrm{P} 4$ position, as even amino acids with $D$-stereochemistry were rapidly hydrolyzed. This screen of a large peptide library using legumain yielded several substrates that were not recognized by caspases. The champion substrate was the tetrapeptide Ac-DTyr-Tic-SerAsp-ACC, which was fairly well hydrolyzed by legumain $\left(k_{\text {cat }} / K_{M}=3970 \mathrm{M}^{-1} \mathrm{~s}^{-1}\right)$ and almost completely ignored by the apoptotic caspases 3, 6, 8, 9 and 10 (Figure 6). Moreover, this substrate was cleaved at a much faster rate by legumain that the substrate with the classical Z-Ala-Ala-Asp motif (Z-AAD-ACC: $k_{\text {cat }} / K_{M}=1600 \mathrm{M}^{-1} \mathrm{~s}^{-1}$ ), indicating that the use of unnatural amino acids in protease substrates significantly increases both their selectivity and activity. Although substrates with unnatural (nonproteinogenic) amino acids cannot be used to predict the cleavage site in natural substrates, they represent a valuable tool for the selective detection of legumain activity in biological samples and can also be used as scaffolds for the development of selective inhibitors and activity-based probes (Poreba et al., 2017a).

\section{Legumain inhibitors}

During the initial characterization of legumain in the 1990s, multiple inhibitors were tested to block the activity of this protease and obtain insights into its catalytic preferences. As is shown in the 1993 study Kembhavi et al., legumain (isolated from Vigna aconitifolia, moth bean) is not inhibited by E-64 and only poorly inhibited by chicken cystatin (Kembhavi et al., 1993). As E-64 and cystatin are both potent and broad spectrum cysteine protease inhibitors, the authors speculated that legumain must differ from other cysteine lysosomal proteases. In the same year, a similar inhibition profile was also described by Abe et al. (1993). Legumain isolated from the seeds of the jack bean (Canavalia ensiformis) is insensitive to E-64 inhibition and only poorly inhibited by various mammalian cystatins. However, in the 1997 study by Chen and coworkers, pig legumain was indeed resistant to E-64, but in contrast to previous reports, this protease was inhibited by nanomolar concentrations of both ovocystatin and human cystatin C (Chen et al., 1997). This characteristic was later confirmed by Johansen et al. (1999). These apparent discrepancies between plant and mammalian proteases are explained by the fact that mammalian cystatins may not be suitable for the inhibition of moth and jack bean legumains. Moreover, serine, aspartic and metalloprotease inhibitors have no effect on legumain activity (Chen et al., 1997). The fact that legumain is only active in the presence of reducing agents allowed assigning this enzyme to the family of cysteine proteases. Although legumain is very labile at neutral $\mathrm{pH}$ and, in this respect, it resembles cysteine cathepsins from the $\mathrm{C} 1$ clan, this protease displays a distinct inhibitory profile, suggesting that legumain may belong to a different clan (Chen et al., 1997). Indeed, in subsequent studies, it the inhibition of legumain by cystatins was shown to differ from the inhibitory mechanism of papainlike enzymes (Alvarez-Fernandez et al., 1999).

Legumain displays a very narrow specificity in the P1 position and thus only mediates the proteolysis of a limited number of substrates; therefore, researchers have speculated that this enzyme might have important biological functions (Rawlings et al., 2018). Based on these characteristics, potent and selective synthetic inhibitors would be invaluable tools for studying legumain biology. These inhibitors are usually designed to closely resemble peptide substrates and as, at that time, legumain was the only enzyme known to hydrolyze substrates after Asn residues, the development of legumain-selective inhibitors appeared to be very straightforward. Unfortunately, inhibitors with Asn in the P1 position are not stable, as they undergo intramolecular cyclization between the amide moiety and $\mathrm{P}^{\prime}$ electrophilic binding group, leading to the loss of inhibitory activity (Figure 7A) (Niestroj et al., 2002). This conclusion was based on the kinetic and structural analysis of Michael acceptor inhibitors containing Asn in the P1 position. The rearrangement of the Asn-warhead, which leads to inactivation, was also observed by Loak et al. in their study of P1-Asn acyloxymethylketones (Loak et al., 2003). Niestroj and coworkers developed a series of azaAsn inhibitors in which the alpha carbon (chiral $\mathrm{CH}$ group) was replaced by a nitrogen to overcome this problem (Niestroj et al., 2002). This change resulted in significantly higher stability and maintained the inhibitory potency towards mammalian legumain. The best inhibitors from this series were Z-Ala-Ala-azaAsn-CMK (chloromethylketone; $k_{\text {obs }} / I$ of $139000 \mathrm{M}^{-1} \mathrm{~s}^{-1}$ ) and Z-AlaAla-azaAsn-BMK (bromomethylketone; $k_{\text {obs }} / I$ of 84000 $\mathrm{M}^{-1} \mathrm{~s}^{-1}$, which both displayed an irreversible mode of inhibition (Figure 7B).

Shortly thereafter, aza-peptide legumain inhibitors with diverse $\mathrm{P}^{\prime}$ leaving groups were also described by the Powers group (Figure 8). In 2002, Asgian et al. synthesized two epoxide-containing Z-Ala-Ala-azaAsn inhibitors that 


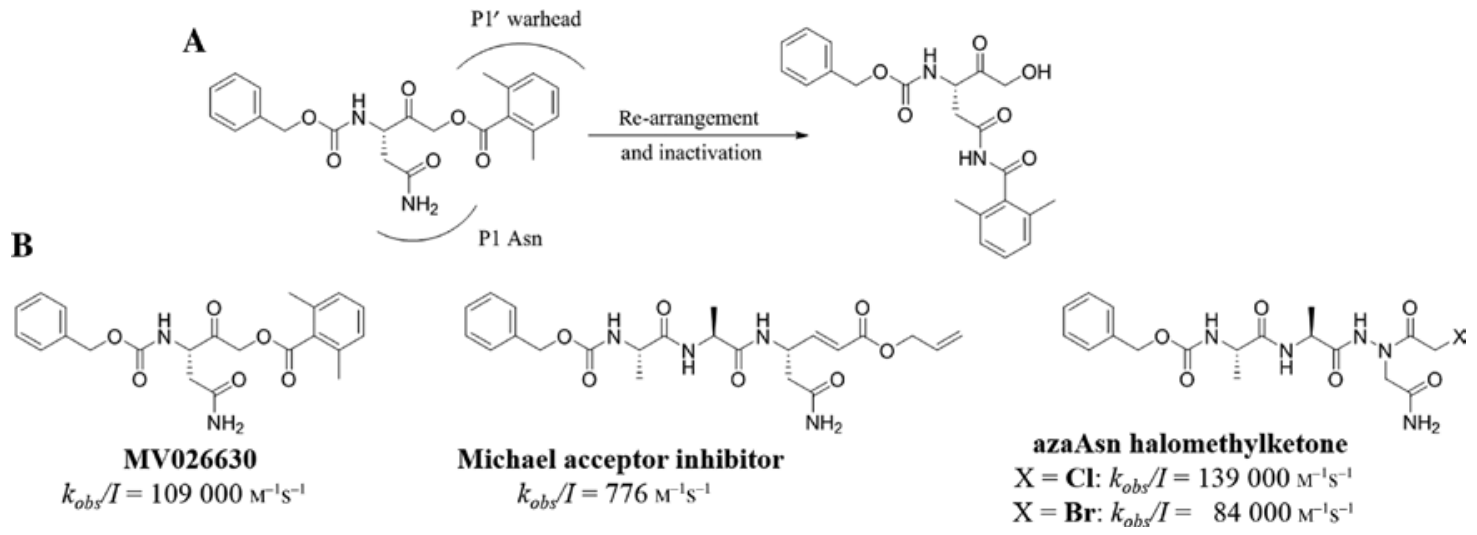

Figure 7: Legumain inhibitors with Asn at P1 position.

(A) Kinetic studies demonstrated that P1-Asn inhibitors are moderately stable, as they undergo intramolecular re-arrangement, which leads to their inactivation. (B) Examples of P1-Asn legumain inhibitors (acyloxymethylketone, Michael acceptor and two halomethylketones). To increase the stability of P1-Asn inhibitors, P1 alpha-carbon was replaced with nitrogen to obtain AZAASN inhibitor.

A<smiles>[R]N(CC(N)=O)NC(=O)C(C)NC(=O)[C@H](C)NC(=O)OCc1ccccc1</smiles>

B<smiles>CC(=O)N1CCN(C(=O)OC(C)(C)C)CC1</smiles>

$I C_{50}=80 \mathrm{nM}($ S.mansoni $)$ $I C_{S_{0}}=8.5 \mathrm{nM}$ (I. ricimis)<smiles>CC(C)C(=O)N1CCN(C(=O)OCc2ccccc2)CC1</smiles>

$I C_{50}=70 \mathrm{nM}($ S.mansoni) $I C_{50}=0.14 \mathrm{nM}$ (I. ricimus)
Epoxides

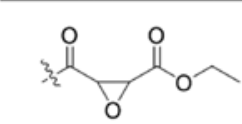

$S, S k_{0, s} / I=43000 \mathrm{M}^{-1} \mathrm{~s}^{-1}$ $R, R k_{o b s} / I=25200 \mathrm{M}^{-1} \mathrm{~s}^{-1}$ (pig kidney legumain)<smiles>CC(=O)C1OC1C(=O)OCc1ccccc1</smiles>

$S, S \quad k_{o b s} / I=26700 \mathrm{M}^{-1} \mathrm{~s}^{-1}$ trans $k_{\text {os }} / I=28900 \mathrm{M}^{-1} \mathrm{~s}^{-1}$ (pig kidney legumain)

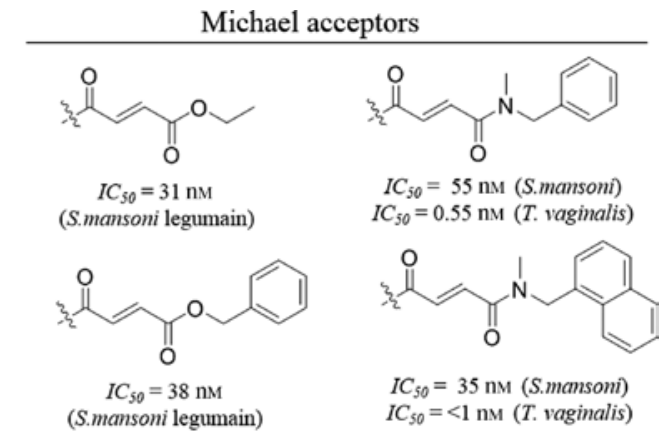

Figure 8: Selected examples of P1-azaAsn legumain inhibitors.

(A) SAR studies to dissect legumain preferences in the prime region using epoxide and Michael acceptor inhibitors with classical Cbz-AlaAla-azaAsn motif. (B) Exploration of P5-P4 legumain preferences with a panel of Michael acceptor inhibitors developed on Ala-Ala-azaAsn$\mathrm{CH}=\mathrm{CH}$-COOET scaffold.

displayed good inhibitory potency towards legumain (S,S isomer: $k_{\text {obs }} / I$ of $43000 \mathrm{M}^{-1} \mathrm{~S}^{-1}$, and R,R isomer: $k_{\mathrm{obs}} / I$ of 25 $\left.200 \mathrm{M}^{-1} \mathrm{~S}^{-1}\right)$ and did not inhibit other proteases from the CA clan (papain and cathepsin B) or the CD clan (caspases 1, 3, 6, and 8) (Figure 8A) (Asgian et al., 2002). As aza-peptide epoxides can be easily extended to explore the protease prime region, the same group reported the synthesis and kinetic analysis of Z-Ala-Ala-azaAsn analogs in a follow-up study (James et al., 2003). Two inhibitors from this series displayed good inhibitory potency towards porcine legumain, providing insights into the structural preferences of this protease for amino acids in the $\mathrm{P} 1^{\prime}$ position. Moreover, when tested against $S$. mansoni parasite legumain and human legumain, these compounds also showed good inhibitory activity, highlighting the mechanistic similarities between parasitic and mammalian legumains. Another 
class of legumain P1 azaAsn inhibitors was discovered by Ekici and coworkers (Figure 8A) (Ekici et al., 2004). The authors synthesized a panel of Z-Ala-Ala-azaAsn-based Michael acceptors with various $\mathrm{P}^{\prime}$ leaving groups that displayed nanomolar affinity for $S$. mansoni legumain $\left(I C_{50}\right.$ from 31 to $55 \mathrm{nM}$ ). A much broader analysis of aza-peptidyl Michael acceptors as legumain inhibitors was conducted in 2008 by Gotz et al., in which the authors reported the design, synthesis and kinetic analysis of a comprehensive set of 31 of this type of inhibitors towards three medically important parasites: Trichomonas vaginalis (a protist), Ixodes ricinus (a hard tick) and the previously described S. mansoni (a flatworm) (Gotz et al., 2008). The kinetic analysis of multiple inhibitors, all of which contained the Z-Ala-Ala-azaAsn scaffold, provided detailed insights into the structural preferences of the active sites parasitic legumains for amino acids in the prime region. These inhibitors display a very wide range of inhibitory potencies towards target enzymes, as the $I C_{50}$ values ranged from 1 to $10000 \mathrm{~nm}$ (Figure 8A). Generally, the SAR analysis revealed that the investigated legumains prefer aromatic residues in the $\mathrm{P}^{\prime}$ ' position, and the orientation and extension of the leaving groups influence the potency and reactivity. More importantly, these inhibitors do not react with other proteases from the $\mathrm{CD}$ (caspases and gingipain) and CA (calpain, papain and cathepsin) clans, highlighting the importance of P1 primary specificity in the development of protease-selective inhibitors. Finally, the authors also dissected the stability of the selected inhibitors in various assay buffers. These inhibitors are generally more stable at $\mathrm{pH} 5.8$ than at $\mathrm{pH} 6.8$, which is of crucial importance for the selective inhibition (and detection) of legumain, which exhibits maximal catalytic activity at $\mathrm{pH}$ values ranging from 5.5 to 6.0. In the next paper from this group, the authors expanded upon their previous development of legumain inhibitors by exploring the $\mathrm{P} 4$ and $\mathrm{P} 5$ positions (Ovat et al., 2009). For this purpose, a set of P5-P4-AlaAla-azaAsn-based tri- and dipeptides and simple azaAsn Michael acceptor and epoxides were synthesized and evaluated against legumains from the parasites I. ricinus and S. mansoni (Figure 8B). Generally, Michael acceptor inhibitors were more potent than epoxides, and the potency within the series increased as the length of the peptide chain increased (tripeptides $>$ dipeptides $>$ mono azaAsn). The most active inhibitors of both S. mansoi and I. ricinus legumain were pentapeptides. In addition to the P4 and P5 analysis, the authors also synthesized several inhibitors with Ala, Val, Ile or Phe in the P2 position, and confirmed that both legumains preferred small amino acids over bulky, hydrophobic amino acids, consistent with the substrate specificity of legumain towards peptide substrates.
Potential aza-peptidyl inhibitors of legumain were also explored by Lee and Bogyo (2012). However, in contrast to other studies focusing on the exploration of the $\mathrm{P}^{\prime}$ position, the authors synthesized a series of Michael acceptor and epoxide inhibitors with different amino acids in the P3 position. The pro-azaAsn dipeptide was used as the scaffold, where proline was incorporated to minimize the cross-reactivity with cysteine cathepsins (Lee and Bogyo, 2010). Moreover, in an effort to increase the potency and selectivity of inhibitors, the authors incorporated various unnatural amino acids in the inhibitor structures. The kinetic analysis revealed that legumain prefers small, alkyl amino acids in the P3 position over bulky groups, and epoxides are slightly more active inhibitors than compounds with a Michael acceptor warhead. Importantly, all tested Michael acceptor inhibitors did not react with lysosomal cathepsin $\mathrm{L}$, whereas epoxides displayed only negligible cross-reactivity. This study highlights the importance of the use of unnatural amino acids in the development of short peptidic inhibitors that distinguish between legumain and other endolysosomal proteases.

Legumain inhibitors with Asn in P1 are not stable, as they slowly undergo intramolecular cyclization (Niestroj et al., 2002). However, as shown in the 2003 study by Loak et al., P1 Asn acyloxymethylketones are very potent $\left(k_{\mathrm{obs}} / I\right.$ for Z-Asn-AOMK is $109000 \mathrm{M}^{-1} \mathrm{~s}^{-1}$ ) and selective (no reactivity with caspases and cathepsins) legumain inhibitors (Loak et al., 2003). In addition, the most potent inhibitor easily enters cells and inhibits endogenous legumain, which was monitored with the Z-Ala-Ala-Asn-AMC substrate and by visualizing of legumain maturation using Western blotting. Although the champion inhibitor was very promising for applications in cell-based studies, its metabolic stability has not been assessed in detail. The synthesis of P1 Asn inhibitors is less problematic than the synthesis of the corresponding P1 azaAsn analogs; thus, these inhibitors represent a very convenient tool to study legumain. However, the moderate stability of these compounds (particularly during storage) is probably the main reason why these compounds are currently less commonly used than azaAsn inhibitors. Thus, other strategies for the development of legumain synthetic inhibitors have been pursued. As this protease also cleaves substrates after Asp, peptides with this amino acid in the P1 position and an electrophile in the $\mathrm{P}^{1}$ position comprise another class of synthetic legumain inhibitors. In 2003, RozmanPungercar and coworkers showed some cross-reactivity between classical CMK- and FMK-based caspase inhibitors (Z-DEVD-cmk, Ac-YVAD-cmk and Z-VAD-fmk) with legumain, and the compounds moderately inhibited this protease (Figure 9) (Rozman-Pungercar et al., 2003). Lee 
20

Z-Val-Ala-Asp-fmk

Legumain $\quad k_{o b s} / I=1200 \mathrm{M}^{-1} \mathrm{~s}^{-1}$ Caspase-3 $k_{o b s} /=6500 \mathrm{M}^{-1} \mathrm{~s}^{-1}$

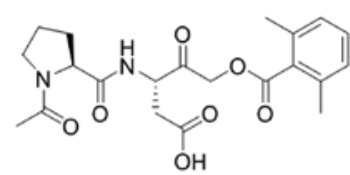

Ac-Pro-Asp-AOMK
Legumain $I C_{50}=0.7 \mathrm{~mm} k_{o b s} /=1900 \mathrm{M}^{-1} \mathrm{~s}^{-1}$ Caspase-3 $I C_{50}=2.8 \mathrm{~mm}$

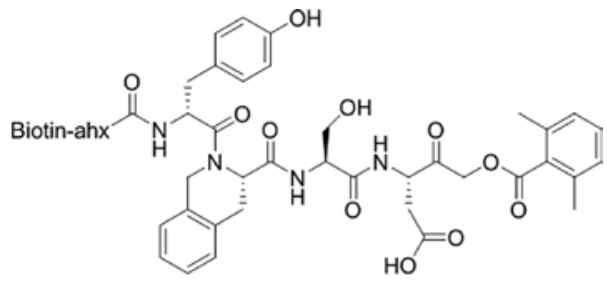

Biotin-ahx-DTyr-Tic-Ser-Asp-AOMK

Legumain $k_{\text {obs }} / I=34000 \mathrm{M}^{-1} \mathrm{~s}^{-1}$

Caspase-3 $k_{o b s} / I \quad<100 \mathrm{M}^{-1} \mathrm{~s}^{-1}$

Figure 9: Selected examples of legumain inhibitors with ASP at the P1 position.

Z-VAD-FMK inhibitor was originally designed for caspases, but also displays cross-reactivity with legumain. AC-PRO-Asp-AOMK displays improved selectivity towards legumain, and biotin-AHX-DTyr-Tic-Ser-Asp-AOMK (containing unnatural amino acids) is almost exclusively recognized by legumain, and shows only negligible reactivity with caspases.

and Bogyo developed a short, dipeptidic Ac-Pro-AspAOMK inhibitor to reduce the off-target activity of P1 Asp inhibitors towards caspases (Lee and Bogyo, 2010, 2012). As caspases require at least four amino acids for efficient hydrolysis (and inhibition) (Poreba et al., 2013), this truncated peptide will not inhibit these proteases. Indeed, the kinetic analysis revealed that Ac-Pro-Asp-AOMK is a more potent inhibitor of legumain than caspase-3; however, the difference in kinetics was not pronounced (IC $C_{50}$ for legumain $=0.70 \mu \mathrm{M}, I C_{50}$ for caspase-3=2.8 $\mu \mathrm{M}$ ) (Figure 9). Optimal legumain peptides were applied to increase the potency of P1 Asp inhibitors towards legumain and to reduce the cross-reactivity with caspases and cathepsins. In one study employing these peptides, Poreba and coworkers used the legumain-selective peptide containing unnatural amino acids to develop peptide-Asp-AOMK (MPL01) that selectively inhibited legumain in a test tube and in living cells and did not cross-react with other proteases (Figure 9) (Poreba et al., 2016). In addition, the authors also synthesized an inhibitor in which P1 Asp was methylated (MP-L02), which is a common strategy to improve cell permeability. Once the methylated compound enters cells, esterases remove the methyl group to activate the inhibitor. Indeed, the methylated inhibitor was taken up by cells faster than its nonmethylated counterpart, but it displayed significantly less potent inhibitory activity, as Asp demethylation proceeded very slowly.

Another class of legumain inhibitors was discovered by the Williams group and described in a series of papers. All these inhibitors contain a cyano electrophilic binding group. The parental compound in this series (Z-Pro-Asn$\left.\mathrm{CN}, I C_{50}=140 \mathrm{nM}\right)$ was identified through a hit development program and further optimized in the P2 and P3 positions (Figure 10, top) (Higgins et al., 2014). In 2014,

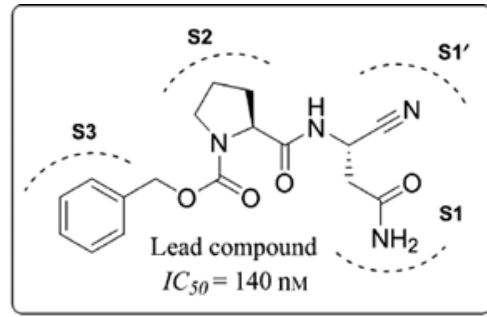

P3 optimization<smiles>CCOc1cccc(-c2ccc(COC(=O)N3CCC[C@H]3C(=O)N[C@@H](C#N)CC(N)=O)cc2)c1</smiles>

P3 and P3-P2 linker optimization<smiles>CC(C)(C)c1ccc(-c2ccc(S(=O)(=O)N3CCCC3C(=O)N[C@@H](C#N)CC(N)=O)cc2)cc1</smiles>

P3-P2 optimization

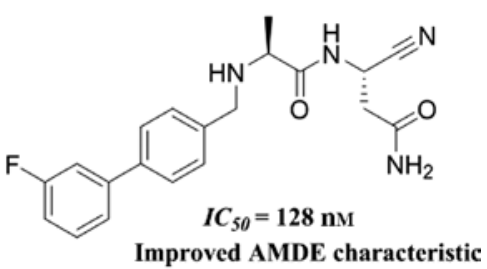

Figure 10: Exploration of P3-P2 legumain preferences with the use of individual libraries of P1-Asn peptidomimetics with cyano (CN) electrophilic binding group. 
Higgins et al. reasoned that as the legumain S2 pocket is shallow and accommodates only small, aliphatic amino acids (alanine, proline and the unnatural amino acid azetidine), increased inhibitory potency and selectivity might be obtained through the optimization of P3-S3 interactions (Higgins et al., 2014). Indeed, the incorporation of an additional phenyl ring into the benzyl group significantly increased the inhibitory potency, indicating that the legumain S3 pocket is deep and can accommodate bulky, hydrophobic moieties. The best compound of this series displayed subnanomolar affinity for legumain $\left(I C_{50}=0.9\right.$ nM) and was 150-fold more active than the lead compound (Figure 10, left). Moreover, this champion inhibitor showed only low to moderate reactivity with selected cathepsins and caspases when tested at a concentration of $30 \mu \mathrm{M}$. Shortly thereafter, the same group aimed to optimize the P2-P3 linker in legumain inhibitor structures (Ness et al., 2016). The use of a sulfonamide group instead of a regular peptide bond significantly increased the inhibitory potency towards legumain. Furthermore, this new lead compound was optimized to explore the chemical space in the legumain S3 pocket by introducing multiple hydrophobic substituents. These SAR efforts resulted in the discovery of several highly potent compounds, which were next tested for their ability to block intracellular legumain activity (Figure 10, middle). In fact, some of the compounds potently inhibited legumain; however, as these inhibitors lack of metabolic stability and display low solubility, they do not appear to be optimal for in vivo applications. Therefore, in a follow-up study, Ness and coworkers aimed to improve physiochemical properties of legumain inhibitors and increase the suitability of these compounds for in vivo studies (Ness et al., 2015). The optimization of the P2-P3 linker resulted in the development of new derivatives that, in contrast to previously reported inhibitors, displayed excellent kinetic solubility and microsomal stability (Figure 10, right). However, as the new inhibitors displayed only submicromolar legumain affinity, additional P2 SAR experiments were performed, but more potent inhibitors were not discovered. This work highlights the importance of maintaining the balance between inhibitor activity and their pharmacokinetic profiles, as even most active and selective compounds might be useful in in vivo studies but display poor ADME-T characteristics.

\section{Legumain activity-based probes}

Legumain inhibitors have been proven to be very useful tools for studying the physiological roles of this enzyme in multiple species. However, these inhibitors are unable to directly visualize this enzyme in biological samples. Activity-based probes (ABPs) are small-molecule inhibitors equipped with a detectable tag; thus, in addition to inhibiting enzymatic activity, ABPs enable the detection of their activity in a tag-dependent manner (Saghatelian et al., 2004; Fonovic and Bogyo, 2008; Sanman and Bogyo, 2014). Currently, the most commonly used tags in ABP designs are fluorophores that enable direct visualization of the enzymes; however, other tags, such as biotin or the ${ }^{125} \mathrm{I}$ radioisotope, are still in use. The first ABPs for legumain were developed by the Bogyo group. In 2005, Kato and coworkers synthesized a panel of P1-AOMK inhibitors labeled with biotin and the ${ }^{125}$ r radioisotope (Figure 11A) (Kato et al., 2005). Two of these probes (with Asp or Asn in the P1 position) inhibited and labeled purified human legumain. While Asp-AOMK displayed more pronounced labeling, only Asn-AOMK was selective for legumain and did not react with caspase-3. Moreover, both probes efficiently labeled endogenous legumain in lysates from 8.1.6 cells, where the Asp-AOMK probe displayed a narrow reactivity window (acidic $\mathrm{pH}$ ), and AsnAOMK was active in the whole $\mathrm{pH}$ range tested $(\mathrm{pH}$ ranging from 3.5 to 6.5). This observation reflects the substrate kinetics, where peptides with Asp are cleaved by legumain only at acidic $\mathrm{pH}$. Although the Asp-AOMK probe efficiently reacted with legumain, this compound lacks cell permeability. Therefore, in 2007, the Bogyo group aimed to develop fluorescently labeled legumain ABPs with improved cell permeability (Sexton et al., 2007). Two prototypic probes with either a single Asp amino acid or Val-Ala-Asp tripeptide reacted with legumain in lysates from 816 B and RAW264.7 cells. However, as these probes were originally developed to target caspases, the authors set out to develop a more legumainselective peptide scaffold. The screen of a combinatorial library of P4-P3-P2-Asp-AOMK inhibitors of legumain yielded the VPED sequence, which distinguished between legumain and caspases. However, the fluorescent probe incorporating the VPED peptide reacts with abundantly expressed cathepsin B. The authors synthesized two truncated probes with ED and PD dipeptides to decrease the reactivity of the ABPs towards cathepsin $B$ and showed that while ED probe still displays cross-reactivity, the PD analogue selectively labeled legumain, even at high concentrations (Figure 11B). Finally, when probes were incubated with living RAW264.7 cells, both showed efficient legumain labeling, but again, only PD ABP was legumainselective and did not react with cathepsin B. This study was the first to reveal selective legumain labeling in living cells using small, fluorescent, activity-based peptide 
A

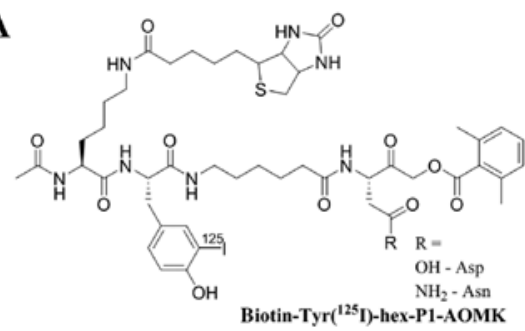

B

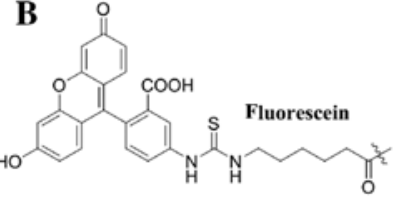

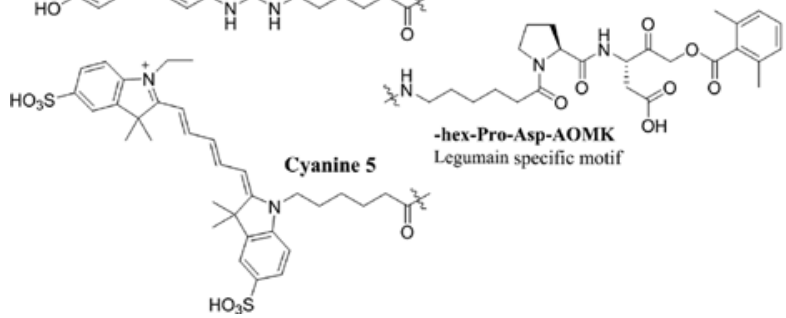

C

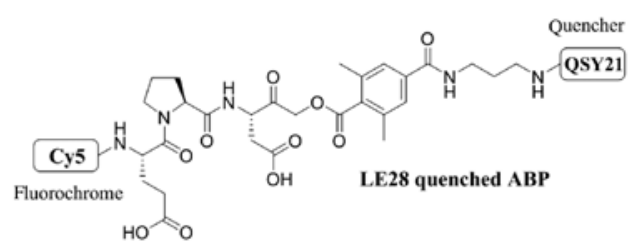

D
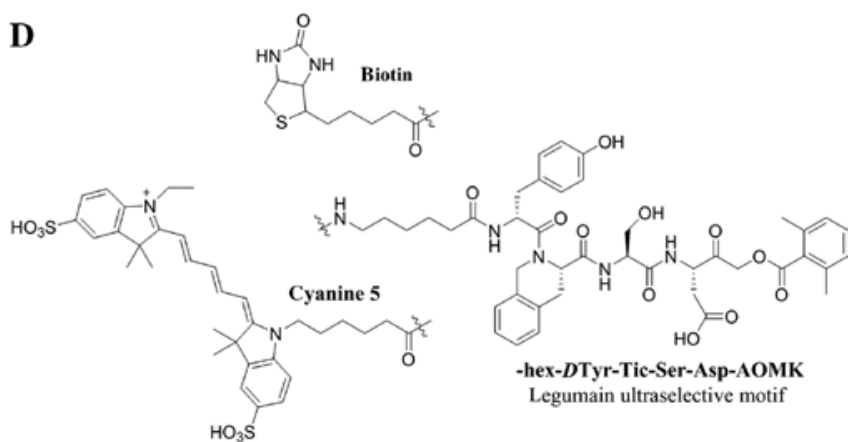

Figure 11: Several structures of P1-Asp activity-based probes (ABPs) for legumain detection.

(A) Prototypic abp with two tags - biotin and Tyr(125)). (B) Two fluorescent ABPs with Pro-Asp-AOMK motif. (C) First quenched activity-based probes for legumain with near infra-red cyanine 5 fluorophore and QSY21 quencher. (D) Two ultraselective ABP for legumain with biotin/ cyanine 5 tags and peptide sequence containing unnatural amino acids.

probes. In another study from the Bogyo lab, the authors utilized the EPD-AOMK motif and labeled it with a Cy5 (cyanine 5) fluorophore to make a covalent ABP targeting both caspases and legumain (called AB50-Cy5) (Edgington et al., 2009). The use of Cy5, which emits at nearinfrared wavelengths, was motivated by the fact that these probes display significantly less background than ABPs decorated with (carboxy)fluorescein. The incubation of this probe with cells undergoing apoptosis resulted in the labeling of both executioner caspases and legumain. However, when the AB50-Cy5 probe was equipped with a penetrating sequence, the probe uptake was remarkably increased and resulted in the more pronounced legumain labeling. As the AB50-Cy5 probe was originally designed for caspases and legumain labeling was only an off-target reaction, Lee and Bogyo next aimed to develop a legumain-tailored Cy5-labeled probe utilizing the optimal peptide sequence (Lee and Bogyo, 2010). Utilizing the previously reported Pro-Asp dipeptide scaffold, the authors synthesized the PD-AOMK inhibitor that was N-terminally labeled with Cy5 fluorophore (LP-0 probe) (Figure 11B). However, since the Pro-Asp sequence is partially recognized by caspases, the authors also developed Cy5-labeled ABP with the Pro-azaAsn-epoxide moiety (LP-1 probe), which was the first P1 Asn activitybased probe reported for legumain (Figure 12A). The incubation of these ABPs with NIH-3T3 and RAW264.7 cells resulted in efficient legumain labeling, with no
A

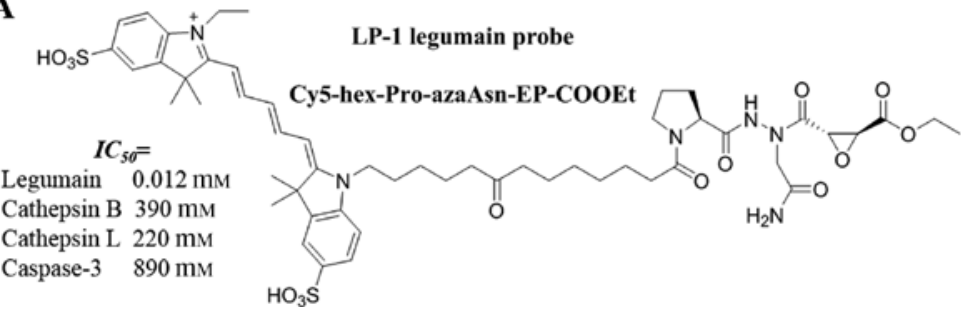

B

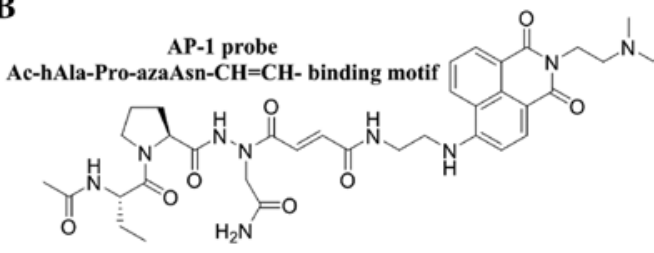

Figure 12: Two examples of legumain ABPs with P1 azaAsn motif.

(A) Fluorescent ABP tagged with cyanine 5 and truncated PRO-azaAsn-epoxide scaffold. (B) Michael acceptor-based ABP in which the fluorescent tag was attached to the C-terminal end of the molecule. Upon binding to legumain, this on-off ABP generates fluorescence through photo-induced electron transfer. 
cross-reactivity with lysosomal cathepsins at low ABP concentrations (10-100 nM). The observation that LP-1 probes displays poor cross-reactivity with cathepsins is consistent with previous findings that aza-peptide inhibitors are not suitable for blocking the activity this group of proteases. Moreover, as the LP-1 ABP containing Asn in the $\mathrm{P} 1$ position was a much more potent probe than the $\mathrm{P} 1$ Asp LP-0 probe, LP-1 ABP was further used for the optical imaging of legumain activity in tumors in live mice. Finally, the authors conjugated the LP-1 probe with various penetrating peptides (Tat, Penetratin and R8) or with cholesterol to increase the cellular uptake of the probe. Unexpectedly, although probes with penetrating peptides displayed enhanced legumain labeling, they also significantly cross-reacted with multiple cathepsins, most likely due to increased lysosomal uptake. The ABP conjugated with cholesterol displayed the weakest crosslabeling with cathepsins, but still it was less selective than the parental LP-1 ABP. Nevertheless, Cy5-labeled azaAsn inhibitors appear to be excellent tools for the selective detection of the recombinant legumain protein and the endogenous legumain protein expressed in living cells and whole organisms. Although fluorescent ABPs are valuable tools for the direct detection of legumain activity, they also display high fluorescent background when used at high concentrations. This characteristic impedes their application in real time in vivo enzyme imaging. The Bogyo group developed smart activitybased probes that only become fluorescent when they react with the target enzymes to overcome this issue (Figure 11C) (Edgington et al., 2009). These compounds, called quenched activity-based probes (qABPs), are composed of a peptidic inhibitor flanked by fluorophore and a quencher moiety, and currently are among most frequently used chemical tools for protease imaging. The mode of the action of these compounds is similar to internally quenched substrates (IQFs); however, in contrast to IQFs, where hydrolysis products diffuse away, in qABPs, the fluorescent product remains attached to the enzyme, allowing for its spatiotemporal detection (Paulick and Bogyo, 2008). In the aforementioned study, the authors used a qABP with an EPD peptide motif to detect legumain activity in cultured cells and live mice (Edgington et al., 2009). Although, the EPD peptide is well recognized by caspases, the specificity towards legumain is mainly driven by the presence of a hydrophobic fluorophore and quencher, which reduce the off-target activity of qABP. Moreover, this probe is taken up by cells via an endocytic pathway and directed to endolysosomal compartments, thus bypassing the cytosol, where the caspases reside. In an attempt to develop truly legumain-selective ABPs that will be insensitive to both caspases and cathepsins, Poreba and coworkers used the HyCoSuL approach to dissect legumain preferences for amino acids in the P4-P2 positions (Poreba et al., 2016). The use of a large panel of unnatural amino acids in this screen resulted in the development of highly selective legumain tetrapeptide substrates that were converted into a biotin-labeled ABP (biotin-ahx-DTyr-Tic-Ser-AspAOMK, MP-L01) (Figure 11D). This probe efficiently labeled legumain in intact living cells, displaying virtually no cross-reactivity with cathepsins. Cells were cotransfected with caspase- 8 and procaspase- 3 to further examine the selectivity of the probe. This approach resulted in the robust activation of caspase-3 (detected by the biotin-ahx-DEVD-AOMK probe), but reactivity with the legumain ABP probe was not detected, even at a high ABP concentration $(1 \mu \mathrm{M})$. In a follow-up study, the biotin tag from MP-L01 was replaced with the Cy5 fluorophore, and the new probe (MP-L09) was used to track and visualize the internalization of active legumain in colon cancer cells (Figure 11D) (Lunde et al., 2017). In conclusion, the discovery of MP-L01 and MP-L09 probes, along with earlier studies conducted by the Bogyo group, indicate that the leveraging of a wide set of unnatural amino acids in the P4-P2 positions enables a truly selective legumain probe to be obtained, even when the P1 position is occupied by caspase-preferred Asp residue.

Recently, another type of inhibitor-based smart probe was developed (Hong et al., 2017). Utilizing previously reported legumain-selective Ac-hAla-Pro-azaAsn peptide, Hong and coworkers synthesized a Michael acceptor inhibitor labeled with a fluorophore (AP-1 probe) that generates a fluorescence response only after reacting with legumain through an effect called photo-induced electron transfer (PeT) (Figure 12B) (Hong et al., 2017). The utility of the AP-1 probe for legumain visualization was confirmed in live cell imaging experiments using various cell types, such as macrophages, fibroblasts, neurons and colorectal cancer cells.

\section{Legumain-activated cancer prodrugs}

Cancer is one of the most devastating diseases, leading to millions deaths per year. The major hallmark of cancer is the abnormal growth of cells that potentially migrate and invade other organs in the body (Hanahan and Weinberg, 2011). The considerable focus on cancer research has yielded several strategies to combat this disease. Most 
cancer therapies rely on the repeated administration of cytotoxic agents that inhibit cancer cell proliferation and induce apoptosis (Pistritto et al., 2016). These chemotherapeutics are very potent; however, their nonselective distribution is associated with high toxicity towards healthy cells. Moreover, based on accumulating evidence, multiple proteases (particularly cathepsins and MMPs) that are expressed at high levels and secreted by TAMs (tumorassociated macrophages) modulate the chemotherapy efficiency (Kessenbrock et al., 2010; Shree et al., 2011; Olson and Joyce, 2013). An alternative approach to combatting cancer is prodrugs, which are currently the fastest growing segment in oncology (Giang et al., 2014; Peters and Brown, 2015). These molecules contain a chemotherapeutic drug (payload) that is conjugated with a chemical moiety that is specifically recognized by an enzyme. In principle, the prodrug displays no or only negligible biological activity in its native state and becomes fully active upon cleavage by an enzyme (Figure 13A) (Rooseboom et al., 2004). Most prodrugs are activated by proteases displaying increased expression in cancer, which significantly reduces their systemic off-target toxicity while maintaining high anticancer efficiency (Figure 13B) (Choi et al., 2012; Hamilton and Trejo, 2017).

The study on legumain substrate specificity has yielded multiple peptides that are exclusively hydrolyzed by this protease. Because legumain is expressed at high levels in multiple cancers, legumain-selective peptides are ideal candidates for the development of legumainactivated prodrugs (Liu et al., 2003). The first report regarding the development of a legumain-activated drug was provided by the Edgington group (Liu et al., 2003). In this proof of concept study, the authors conjugated the anticancer drug doxorubicin with the legumain-selective Boc-Ala-Ala-Asn-Leu peptide to obtain Boc-Ala-Ala-AsnLeu-doxorubicin, which was called legubicin (Figure 14A). In this prodrug, legumain cleaves the peptide bond between Asn and Leu to release Leu-doxorubicin, which is further converted into the tumoricidal end product doxorubicin. This compound displays remarkably lower toxicity towards wild-type 293 cells than nonconjugated doxorubicin, and is equally potent (cytotoxic) when these cells were transfected with legumain, suggesting that its antitumor activity depended on the intracellular legumain activity. However, as legumain displays extracellular activity in the tumor microenvironment of multiple cancers, cell-impermeable legumain-activated prodrugs appear to be very valuable therapeutics, as they kill both cancer and cancer-associated cells through the so-called bystander effect. In an attempt to achieve this goal, $\mathrm{Wu}$ and coworkers synthesized a panel of legubicin derivatives in which the prodrugs were N-terminally capped with a succinyl group that significantly decreases the cell permeability of the cargo molecule (Figure 14B) (Wu et al., 2006). Among this series, the LEG-3 prodrug (succinyl-Thr-Ala-Asn-Leu-doxorubicin) was a highly potent inhibitor in legumain-overexpressing 293 cells. The LEG-3 prodrug also displayed decreased levels of overall toxicity ( $>10$-fold) and cardiotoxicity (>15-fold) in mice compared to doxorubicin and high antitumorigenic potency when administered to mice bearing CT26 and C1300 tumors, resulting in a markedly higher survival rate in mice. In subsequent studies, LEG-3 significantly decreased the number of tumor-associated macrophages, circulating tumor cells (CTCs) and myeloid-derived suppressor cells (MDSCs) in mice models. Moreover, this prodrug displayed
$\mathbf{A}$

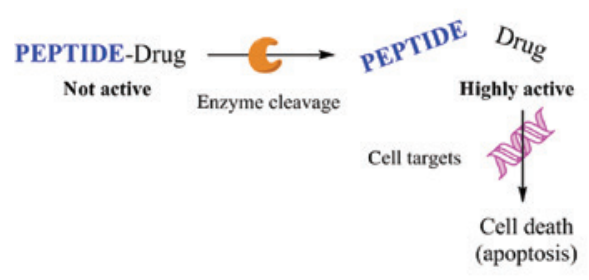

B

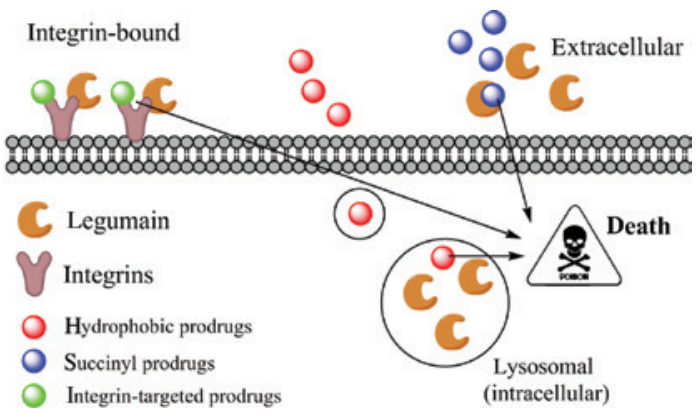

Figure 13: The principles of protease-activated prodrugs.

(A) Simplified prodrug architecture. A chemotherapeutic agent (drug, payload) is conjugated to protease-sensitive peptide. Most prodrugs display almost no biological activity. Upon proteolysis by target enzyme, drug is released to induce cell death (mainly through apoptosis). (B) Legumain-activated prodrugs can be divided into three main groups: (1) prodrugs targeting intracellular, lysosomal legumain; prodrugs are taken up via endocytosis/pinocytosis, (2) prodrugs targeting extracellular legumain; after prodrugs activation, free drug is internalized by the cell, (3) prodrugs targeting integrins which are known to bind extracellular legumain; upon binding to integrins and cleavage by legumain, free prodrug is taken up by cells. 
A

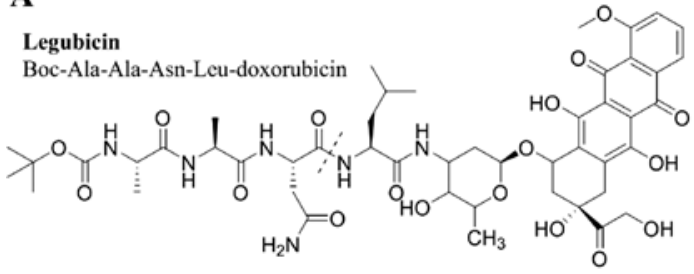

C

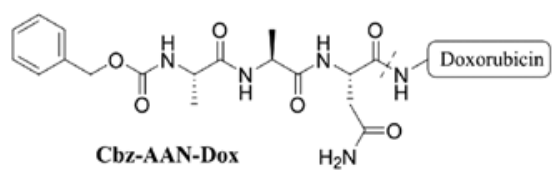

B

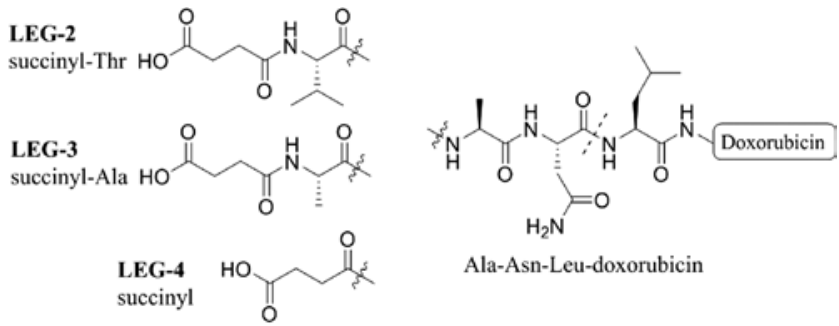

D

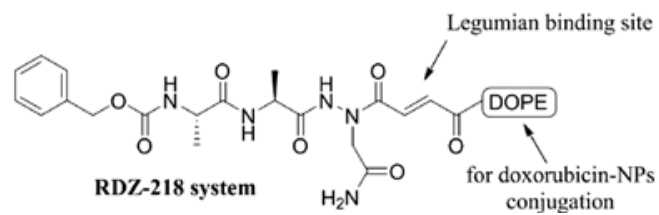

Figure 14: Examples of substrate- and inhibitor-based legumain-activated prodrugs with doxorubicin chemotherapeutic. (A) Legubicin - a prototypic substrate-based legumain prodrug with Boc-Ala-Ala-Asn peptide and Leu-doxorubicin drug. (B) Three doxorubicin-based prodrugs with various P4-P3 substituents. (C) Legumain prodrug in which enzyme recognition sequence (Cbz-Ala-AlaAsn) was directly coupled to doxorubicin. (D) RDZ-218, an inhibitor-based legumain prodrug, which binds to the legumain in an irreversible fashion.

marked antimetastatic activity in a lung cancer model. The high therapeutic efficiency of LEG-3 in various tumor models, together with its safe toxicity profile, shows that the development of legumain-selective cell-impermeable prodrugs appears to be a promising strategy to combat cancer. In another study, Chen and coworkers synthesized the Cbz-Ala-Ala-Asn-doxorubicin prodrug, where the legumain cleavage sequence was directly attached to the doxorubicin moiety (Figure 14C) (Chen et al., 2015). Based on the results of the in vitro analysis with purified legumain, legumain cleaves the prodrug between Asn and doxorubicin; however, this hydrolysis is incomplete, even after $24 \mathrm{~h}$. Nevertheless, this prodrug inhibits the growth of and induce apoptosis in several cancer cell lines. An interesting approach utilizing doxorubicin and a legumain-targeted peptide was described by Liao and colleagues (Liao et al., 2011). In contrast to other studies, where substratebased legumain prodrugs were synthesized, the authors developed a new type of a prodrug which is an inhibitor. Cbz-Ala-Ala-Asn-Michael acceptor, a potent legumain inhibitor was coupled to a DOPE molecule, which is a component of liposomal NPs. Next, these NPs were loaded with the doxorubicin cargo to obtain RDZ-218 (Figure 14D). This inhibitor, once bound to cell surface legumain, is irreversibly internalized along with the enzyme into the target cell, where doxorubicin is released from targeted nanoparticles to induce cell death. The anticancer activity of this tumor-homing system was confirmed in vivo, as the RDZ-218 treatment significantly decreased the tumor weight. Moreover, the RDZ-218 therapeutic agent is markedly less toxic that free doxorubicin and nanoparticlecoated doxorubicin.
According to the results of aforementioned studies, doxorubicin-containing substrate- or inhibitor-based prodrugs are promising strategies for legumain-assisted cancer drug delivery and release. Thus, in further research efforts, various types of chemotherapeutics were also investigated in this context. One of these compounds is etoposide, which intercalates into DNA and inhibits the topoisomerase II enzyme, similar to doxorubicin (Karpinich et al., 2002). In 2009, Stern and colleagues developed a legumain-selective prodrug with the Cbz-Ala-Ala-Asn-EDAetoposide structure, where the peptide was conjugated to etoposide through an ethylenediamine (EDA) linker (Figure 15A) (Stern et al., 2009). This linker was introduced to facilitate legumain-assisted prodrug hydrolysis and generate the EDA-etoposide fragment, which in turn undergoes cyclization at a neutral cytosolic $\mathrm{pH}$, releasing the free drug. These self-immolative linkers are currently widely used in the design of multiple prodrugs. This prodrug was efficiently hydrolyzed by both purified and endogenous legumain. Next, the prodrug and free etoposide were tested in HEK293 and legumain-overexpressing HEK293 cells to assess its biological utility. While etoposide inhibited the proliferation of both cell lines, Cbz-AlaAla-Asn-EDA-etoposide showed no activity towards these cells. The failure of the prodrug to kill legumain-overexpressing HEK293 cells is most likely explained by the observation that the EDA-etoposide cleavage product was not able to translocate from lysosomes to cytoplasm and thus did not undergo cyclization to release free etoposide. Thus, further efforts to optimize the linker technology are needed. Another drug that was applied as a legumaincleavable prodrug was monomethyl auristatin E (MMAE) 


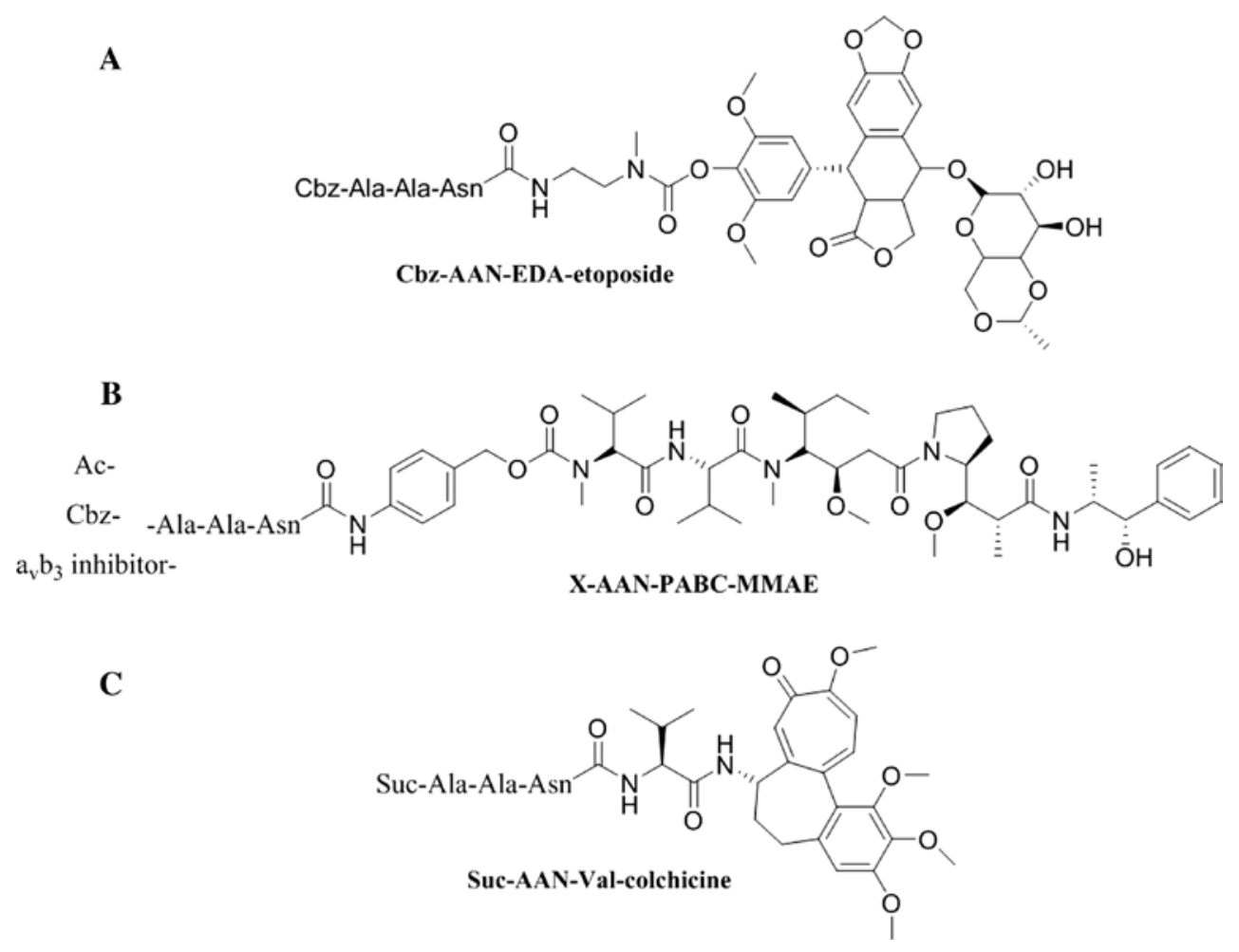

Figure 15: Examples of legumain prodrugs with various payload.

(A) Etoposide, (B) monomethyl auristatin E, and (C) colchicine.

(Figure 15B) (Bajjuri et al., 2011; Liu et al., 2012). MMAE is a very potent antimitotic agent, and in contrast to doxorubicin and etoposide, it must only be administered in the prodrug form as it displays significant, nonselective toxicity towards healthy cells (Singh et al., 2008). In 2010, Bajjuri and coworkers reported the development of a CbzAla-Ala-Asn-PABC-MMAE prodrug, where the peptide was conjugated with the drug through a self-immolative para-aminobenzoylcarbamate (PABC) linker (Figure 15B) (Bajjuri et al., 2011). Upon proteolytic activation, PABCMMAE undergoes a 1,6-elimination reaction to release free MMAE. The cytotoxicity studies using cultured cells revealed that, while free MMAE is toxic to all tested cell lines, Cbz-Ala-Ala-Asn-PABC-MMAE only induces apoptosis in cells with high legumain expression and is not toxic to the 4T1 line, which expresses a low amount of this protease. Moreover, the MMAE prodrug was efficient in 4T1 tumor-bearing mice models, where it reduced the tumor volume and markedly decreased the number of lung metastases. In a follow-up study, the same group developed a legumain-targeted MMAE prodrug that was $\mathrm{N}$-terminally capped with an avb3-integrin binding motif (Figure 15B) (Liu et al., 2012). This prodrug was targeted cell surface legumain that is frequently bound to integrins. Indeed, upon a short incubation with the cultured cells, the dual targeted prodrug was more potent towards
MDA-MB-435 cells and less toxic towards HEK293 cells than both the parental Cbz-protected prodrug and nonconjugated MMAE. The high anticancer efficiency of the dual targeted prodrug after a short incubation supported the hypothesis that this compound rapidly binds to integrins on the cell surface and undergoes internalization, followed by legumain-mediated activation and release of the free MMAE drug. The antitumor efficiency of the integrin- and legumain-targeting prodrug was verified in mice models, where this therapeutic inhibited $4 \mathrm{~T} 1$ and D121 lung metastases and reduced tumor volumes in the MDA-MB-435 tumor model. Overall, the targeting of extracellular legumain in the tumor microenvironment with integrin-binding motifs appears to be a good therapeutic approach for multiple cancer types. Another chemotherapeutic agent that was used to design legumain-activated prodrugs is colchicine (Smith et al., 2014), which induces apoptosis by inhibiting microtubule polymerization (Ghawanmeh et al., 2018). In 2014, Smith and coworkers reported the development of the succinyl-Ala-Ala-AsnVal-colchicine prodrug, where a valine was introduced to facilitate prodrug hydrolysis and increase the lipophilicity of the released cleavage product (Figure 15C) (Smith et al., 2014). According to the results of the kinetic analysis, the prodrug is indeed hydrolyzed between Asn and Val to release Val-colchicine. In a study employing cell 
cultures, colchicine was equally toxic to HEK293 and legumain-overexpressing ML38 cells, whereas the prodrug was significantly more active towards the latter cell line. Moreover, when cells were treated with cystatin E/M, a potent legumain inhibitor, the colchicine prodrug displayed less antiproliferative activity. Finally, the prodrug is more potent in HCT116 cells expressing prolegumain and mature legumain than in SW620 cells expressing only proform of this protease.

\section{Summary}

Beginning with the initial discovery and characterization of legumain from moth bean in the early 1990s, this field has witnessed substantial progress in the contexts of both elucidating the roles of legumain in health and disease, as well as developing selective chemical tools to detect and visualize this protease. Legumain plays important roles in multiple physiological processes, and, when dysregulated, contributes to the progression of several diseases, with cancer being the most prominent example. This research was undoubtedly facilitated by the use of legumain-selective substrates, inhibitors and activity-based probes, which confirmed the contributions of legumain to multiple cellular events. Substrates and activity-based probes that are suitable for the direct detection of active legumain may serve as reliable cancer markers in the future, as legumain activity correlates with the prognosis of multiple cancers. On the other hand, approaches targeting the activity of legumain with potent and specific active site inhibitors appear to be a promising anticancer strategy, as extracellularly secreted legumain contributes to matrix remodeling, which in turn leads to cancer cell metastasis. Finally, targeted prodrugs that are selectively activated by intra- and extracellular legumain may also represent valuable tools in the management of cancer and other legumain-associated diseases. Although none of the described tools are currently used in clinical practice, the preclinical data strongly indicates that legumain, similar to other lysosomal proteases, has significant therapeutic potential, and thus further research efforts in translational medicine should be pursued.

Acknowledgments: This project has received funding from the European Union's Horizon 2020 research and innovation programme under the Marie SkłodowskaCurie grant agreement no. 661187, Funder Id: http:// dx.doi.org/10.13039/100010665 and from National Science
Centre in Poland (grant OPUS, UMO-2018/29/B/NZ102249), Funder Id: http://dx.doi.org/10.13039/501100004281.

\section{References}

Abe, Y., Shirane, K., Yokosawa, H., Matsushita, H., Mitta, M., Kato, I., and Ishii, S. (1993). Asparaginyl endopeptidase of jack bean seeds. Purification, characterization, and high utility in protein sequence analysis. J. Biol. Chem. 268, 3525-3529.

Ally, N., Whisstock, J.C., Sieprawska-Lupa, M., Potempa, J., Le Bonniec, B.F., Travis, J., and Pike, R.N. (2003). Characterization of the specificity of arginine-specific gingipains from Porphyromonas gingivalis reveals active site differences between different forms of the enzymes. Biochemistry 42 , 11693-11700.

Alvarez-Fernandez, M., Barrett, A.J., Gerhartz, B., Dando, P.M., Ni, J., and Abrahamson, M. (1999). Inhibition of mammalian legumain by some cystatins is due to a novel second reactive site. J. Biol. Chem. 274, 19195-19203.

Asgian, J.L., James, K.E., Li, Z.Z., Carter, W., Barrett, A.J., Mikolajczyk, J., Salvesen, G.S., and Powers, J.C. (2002). Azapeptide epoxides: a new class of inhibitors selective for clan CD cysteine proteases. J. Med. Chem. 45, 4958-4960.

Bajjuri, K.M., Liu, Y., Liu, C., and Sinha, S.C. (2011). The legumain protease-activated auristatin prodrugs suppress tumor growth and metastasis without toxicity. ChemMedChem 6, 54-59.

Basurto-Islas, G., Grundke-Iqbal, I., Tung, Y.C., Liu, F., and Iqbal, K. (2013). Activation of asparaginyl endopeptidase leads to Tau hyperphosphorylation in Alzheimer disease. J. Biol. Chem. 288, 17495-17507.

Bernath-Levin, K., Nelson, C., Elliott, A.G., Jayasena, A.S., Millar, A.H., Craik, D.J., and Mylne, J.S. (2015). Peptide macrocyclization by a bifunctional endoprotease. Chem. Biol. 22, 571-582.

Briggs, J.J., Haugen, M.H., Johansen, H.T., Riker, A.I., Abrahamson, M., Fodstad, O., Maelandsmo, G.M., and Solberg, R. (2010). Cystatin E/M suppresses legumain activity and invasion of human melanoma. BMC Cancer 10, 17.

Brix, K., McInnes, J., Al-Hashimi, A., Rehders, M., Tamhane, T., and Haugen, M.H. (2015). Proteolysis mediated by cysteine cathepsins and legumain-recent advances and cell biological challenges. Protoplasma 252, 755-774.

Chen, J.M., Dando, P.M., Rawlings, N.D., Brown, M.A., Young, N.E., Stevens, R.A., Hewitt, E., Watts, C., and Barrett, A.J. (1997). Cloning, isolation, and characterization of mammalian legumain, an asparaginyl endopeptidase. J. Biol. Chem. 272, 8090-8098.

Chen, J.M., Rawlings, N.D., Stevens, R.A., and Barrett, A.J. (1998). Identification of the active site of legumain links it to caspases, clostripain and gingipains in a new clan of cysteine endopeptidases. FEBS Lett. 441, 361-365.

Chen, Y.J., Wu, S.C., Chen, C.Y., Tzou, S.C., Cheng, T.L., Huang, Y.F., Yuan, S.S., and Wang, Y.M. (2014). Peptide-based MRI contrast agent and near-infrared fluorescent probe for intratumoral legumain detection. Biomaterials 35, 304-315.

Chen, H., Liu, X., Clayman, E.S., Shao, F., Xiao, M., Tian, X., Fu, W., Zhang, C., Ruan, B., Zhou, P., et al. (2015). Synthesis and evaluation of a CBZ-AAN-Dox prodrug and its in vitro effects on $\mathrm{SiHa}$ cervical cancer cells under hypoxic conditions. Chem. Biol. Drug Des. 86, 589-598. 
Cheng, T., Hitomi, K., van Vlijmen-Willems, I.M., de Jongh, G.J., Yamamoto, K., Nishi, K., Watts, C., Reinheckel, T., Schalkwijk, J., and Zeeuwen, P.L. (2006). Cystatin M/E is a high affinity inhibitor of cathepsin $V$ and cathepsin $L$ by a reactive site that is distinct from the legumain-binding site. A novel clue for the role of cystatin M/E in epidermal cornification. J. Biol. Chem. 281, 15893-15899.

Choi, K.Y., Swierczewska, M., Lee, S., and Chen, X. (2012). Proteaseactivated drug development. Theranostics 2, 156-178.

Cole, P.W., Murakami, K., and Inagami, T. (1971). Specificity and mechanism of clostripain catalysis. Biochemistry 10, 42464252.

Dall, E. and Brandstetter, H. (2012). Activation of legumain involves proteolytic and conformational events, resulting in a contextand substrate-dependent activity profile. Acta Crystallogr. F Struct. Biol. Cryst. Commun. 68, 24-31.

Dall, E. and Brandstetter, H. (2013). Mechanistic and structural studies on legumain explain its zymogenicity, distinct activation pathways, and regulation. Proc. Natl. Acad. Sci. USA 110, 10940-10945.

Dall, E. and Brandstetter, H. (2016). Structure and function of legumain in health and disease. Biochimie 122, 126-150.

Dall, E., Fegg, J.C., Briza, P., and Brandstetter, H. (2015). Structure and mechanism of an aspartimide-dependent peptide ligase in human legumain. Angew. Chem. Int. Ed. 54, 2917-2921.

Dall, E., Hollerweger, J.C., Dahms, S.O., Cui, H., Haussermann, K., and Brandstetter, H. (2018). Structural and functional analysis of cystatin E reveals enzymologically relevant dimer and amyloid fibril states. J. Biol. Chem. 293, 13151-13165.

Dalton, J.P. and Brindley, P.J. (1998). Handbook of Proteolytic Enzymes. Barrett, A.J., Rawlings, N.D., Woessner, J.F., eds, (London, UK: Academic Press), pp. 749-754.

Dando, P.M., Fortunato, M., Smith, L., Knight, C.G., McKendrick, J.E., and Barrett, A.J. (1999). Pig kidney legumain: an asparaginyl endopeptidase with restricted specificity. Biochem. J. 339, 743-749.

Edgington, L.E., Berger, A.B., Blum, G., Albrow, V.E., Paulick, M.G., Lineberry, N., and Bogyo, M. (2009). Noninvasive optical imaging of apoptosis by caspase-targeted activity-based probes. Nat. Med. 15, 967-973.

Ekici, O.D., Gotz, M.G., James, K.E., Li, Z.Z., Rukamp, B.J., Asgian, J.L., Caffrey, C.R., Hansell, E., Dvorak, J., McKerrow, J.H., et al. (2004). Aza-peptide Michael acceptors: a new class of inhibitors specific for caspases and other clan CD cysteine proteases. J. Med. Chem. 47, 1889-1892.

Fonovic, M. and Bogyo, M. (2008). Activity-based probes as a tool for functional proteomic analysis of proteases. Expert Rev. Proteomics 5, 721-730.

Ghawanmeh, A.A., Chong, K.F., Sarkar, S.M., Bakar, M.A., Othaman, R., and Khalid, R.M. (2018). Colchicine prodrugs and codrugs: chemistry and bioactivities. Eur. J. Med. Chem. 144, 229-242.

Giang, I., Boland, E.L., and Poon, G.M. (2014). Prodrug applications for targeted cancer therapy. AAPS J. 16, 899-913.

Gillon, A.D., Saska, I., Jennings, C.V., Guarino, R.F., Craik, D.J., and Anderson, M.A. (2008). Biosynthesis of circular proteins in plants. Plant J. 53, 505-515.

Gotz, M.G., James, K.E., Hansell, E., Dvorak, J., Seshaadri, A., Sojka, D., Kopacek, P., McKerrow, J.H., Caffrey, C.R., and Powers, J.C. (2008). Aza-peptidyl Michael acceptors. A new class of potent and selective inhibitors of asparaginyl endopeptidases (legu- mains) from evolutionarily diverse pathogens. J. Med. Chem. 51, 2816-2832.

Guo, P., Zhu, Z., Sun, Z., Wang, Z., Zheng, X., and Xu, H. (2013). Expression of legumain correlates with prognosis and metastasis in gastric carcinoma. PLoS One 8, e73090.

Halfon, S., Patel, S., Vega, F., Zurawski, S., and Zurawski, G. (1998). Autocatalytic activation of human legumain at aspartic acid residues. FEBS Lett. 438, 114-118.

Hamilton, J.R. and Trejo, J. (2017). Challenges and opportunities in protease-activated receptor drug development. Annu. Rev. Pharmacol. Toxicol. 57, 349-373.

Hanahan, D. and Weinberg, R.A. (2011). Hallmarks of cancer: the next generation. Cell 144, 646-674.

Hara-Nishimura, I., Takeuchi, Y., and Nishimura, M. (1993). Molecular characterization of a vacuolar processing enzyme related to a putative cysteine proteinase of Schistosoma mansoni. Plant Cell 5, 1651-1659.

Haugen, M.H., Johansen, H.T., Pettersen, S.J., Solberg, R., Brix, K., Flatmark, K., and Maelandsmo, G.M. (2013). Nuclear legumain activity in colorectal cancer. PLoS One 8, e52980.

Haugen, M.H., Boye, K., Nesland, J.M., Pettersen, S.J., Egeland, E.V., Tamhane, T., Brix, K., Maelandsmo, G.M., and Flatmark, K. (2015). High expression of the cysteine proteinase legumain in colorectal cancer - implications for therapeutic targeting. Eur. J. Cancer 51, 9-17.

Higgins, C., Bouazzaoui, S., Gaddale, K., D’Costa, Z., Templeman, A., O'Rourke, M., Young, A., Scott, C., Harrison, T., Mullan, P., et al. (2014). P3 SAR exploration of biphenyl carbamate based Legumain inhibitors. Bioorg. Med. Chem. Lett. 24, 2521-2524.

Hong, J.A., Choi, N.E., La, Y.K., Nam, H.Y., Seo, J., and Lee, J. (2017). Development of a smart activity-based probe to detect subcellular activity of asparaginyl endopeptidase in living cells. Org. Biomol. Chem. 15, 8018-8022.

Ishii, S. (1993). [Asparaginylendopeptidase: an enzyme probably responsible to post-translational proteolysis and transpeptidation of proconcanavalin A]. Seikagaku 65, 185-189.

Jackson, M.A., Gilding, E.K., Shafee, T., Harris, K.S., Kaas, Q., Poon, S., Yap, K., Jia, H., Guarino, R., Chan, L.Y., et al. (2018). Molecular basis for the production of cyclic peptides by plant asparaginyl endopeptidases. Nat. Commun. 9, 2411.

James, K.E., Gotz, M.G., Caffrey, C.R., Hansell, E., Carter, W., Barrett, A.J., McKerrow, J.H., and Powers, J.C. (2003). Aza-peptide epoxides: potent and selective inhibitors of Schistosoma mansoni and pig kidney legumains (asparaginyl endopeptidases). Biol. Chem. 384, 1613-1618.

Johansen, H.T., Knight, C.G., and Barrett, A.J. (1999). Colorimetric and fluorimetric microplate assays for legumain and a staining reaction for detection of the enzyme after electrophoresis. Anal. Biochem. 273, 278-283.

Karpinich, N.O., Tafani, M., Rothman, R.J., Russo, M.A., and Farber, J.L. (2002). The course of etoposide-induced apoptosis from damage to DNA and p53 activation to mitochondrial release of cytochrome c. J. Biol. Chem. 277, 16547-16552.

Kato, D., Boatright, K.M., Berger, A.B., Nazif, T., Blum, G., Ryan, C., Chehade, K.A., Salvesen, G.S., and Bogyo, M. (2005). Activitybased probes that target diverse cysteine protease families. Nat. Chem. Biol. 1, 33-38.

Kato, Y., Ozawa, S., Miyamoto, C., Maehata, Y., Suzuki, A., Maeda, T., and Baba, Y. (2013). Acidic extracellular microenvironment and cancer. Cancer Cell Int. 13, 89. 
Kembhavi, A.A., Buttle, D.J., Knight, C.G., and Barrett, A.J. (1993). The two cysteine endopeptidases of legume seeds: purification and characterization by use of specific fluorometric assays. Arch. Biochem. Biophys. 303, 208-213.

Kessenbrock, K., Plaks, V., and Werb, Z. (2010). Matrix metalloproteinases: regulators of the tumor microenvironment. Cell 141, 52-67.

Klinkert, M.Q., Felleisen, R., Link, G., Ruppel, A., and Beck, E. (1989). Primary structures of Sm31/32 diagnostic proteins of Schistosoma mansoni and their identification as proteases. Mol. Biochem. Parasitol. 33, 113-122.

Kuroyanagi, M., Nishimura, M., and Hara-Nishimura, I. (2002). Activation of Arabidopsis vacuolar processing enzyme by selfcatalytic removal of an auto-inhibitory domain of the C-terminal propeptide. Plant Cell Physiol. 43, 143-151.

Lee, J. and Bogyo, M. (2010). Development of near-infrared fluorophore (NIRF)-labeled activity-based probes for in vivo imaging of legumain. ACS Chem. Biol. 5, 233-243.

Lee, J., and Bogyo, M. (2012). Synthesis and evaluation of aza-peptidyl inhibitors of the lysosomal asparaginyl endopeptidase, legumain. Bioorg. Med. Chem. Lett. 22, 1340-1343.

Li, N., Liu, Q., Su, Q., Wei, C., Lan, B., Wang, J., Bao, G., Yan, F., Yu, Y., Peng, B., et al. (2013). Effects of legumain as a potential prognostic factor on gastric cancers. Med. Oncol. 30, 621.

Liao, D., Liu, Z., Wrasidlo, W., Chen, T., Luo, Y., Xiang, R., and Reisfeld, R.A. (2011). Synthetic enzyme inhibitor: a novel targeting ligand for nanotherapeutic drug delivery inhibiting tumor growth without systemic toxicity. Nanomedicine 7, 665-673.

Lin, Y., Wei, C., Liu, Y., Qiu, Y., Liu, C., and Guo, F. (2013). Selective ablation of tumor-associated macrophages suppresses metastasis and angiogenesis. Cancer Sci. 104, 1217-1225.

Liu, C., Sun, C., Huang, H., Janda, K., and Edgington, T. (2003). Overexpression of legumain in tumors is significant for invasion/metastasis and a candidate enzymatic target for prodrug therapy. Cancer Res. 63, 2957-2964.

Liu, Y., Bajjuri, K.M., Liu, C., and Sinha, S.C. (2012). Targeting cell surface alpha(v)beta(3) integrin increases therapeutic efficacies of a legumain protease-activated auristatin prodrug. Mol. Pharm. 9, 168-175.

Loak, K., Li, D.N., Manoury, B., Billson, J., Morton, F., Hewitt, E., and Watts, C. (2003). Novel cell-permeable acyloxymethylketone inhibitors of asparaginyl endopeptidase. Biol. Chem. 384, 1239-1246.

Lunde, N.N., Haugen, M.H., Bodin Larsen, K.B., Damgaard, I., Pettersen, S.J., Kasem, R., Rut, W., Drag, M., Poreba, M., Johansen, H.T., et al. (2017). Glycosylation is important for legumain localization and processing to active forms but not for cystatin E/M inhibitory functions. Biochimie 139, 27-37.

Maehr, R., Hang, H.C., Mintern, J.D., Kim, Y.M., Cuvillier, A., Nishimura, M., Yamada, K., Shirahama-Noda, K., HaraNishimura, I., and Ploegh, H.L. (2005). Asparagine endopeptidase is not essential for class II MHC antigen presentation but is required for processing of cathepsin $L$ in mice. J. Immunol. 174, 7066-7074.

Mai, C.W., Chung, F.F., and Leong, C.O. (2017). Targeting legumain as a novel therapeutic strategy in cancers. Curr. Drug. Targets 18 , 1259-1268.

Manoury, B., Hewitt, E.W., Morrice, N., Dando, P.M., Barrett, A.J., and Watts, C. (1998). An asparaginyl endopeptidase processes a microbial antigen for class II MHC presentation. Nature 396, 695-699.
Manoury, B., Mazzeo, D., Fugger, L., Viner, N., Ponsford, M., Streeter, H., Mazza, G., Wraith, D.C., and Watts, C. (2002). Destructive processing by asparagine endopeptidase limits presentation of a dominant T cell epitope in MBP. Nat. Immunol. 3, 169-174.

Mathieu, M.A., Bogyo, M., Caffrey, C.R., Choe, Y., Lee, J., Chapman, H., Sajid, M., Craik, C.S., and McKerrow, J.H. (2002). Substrate specificity of schistosome versus human legumain determined by P1-P3 peptide libraries. Mol. Biochem. Parasitol. 121, 99-105.

Matthews, S.P., Werber, I., Deussing, J., Peters, C., Reinheckel, T., and Watts, C. (2010). Distinct protease requirements for antigen presentation in vitro and in vivo. J. Immunol. 184, 2423-2431.

Mylne, J.S., Colgrave, M.L., Daly, N.L., Chanson, A.H., Elliott, A.G., McCallum, E.J., Jones, A., and Craik, D.J. (2011). Albumins and their processing machinery are hijacked for cyclic peptides in sunflower. Nat. Chem. Biol. 7, 257-259.

Mylne, J.S., Chan, L.Y., Chanson, A.H., Daly, N.L., Schaefer, H., Bailey, T.L., Nguyencong, P., Cascales, L., and Craik, D.J. (2012). Cyclic peptides arising by evolutionary parallelism via asparaginyl-endopeptidase-mediated biosynthesis. Plant Cell 24, 2765-2778.

Ness, K.A., Eddie, S.L., Higgins, C.A., Templeman, A., D’Costa, Z., Gaddale, K.K., Bouzzaoui, S., Jordan, L., Janssen, D., Harrison, T., et al. (2015). Development of a potent and selective cell penetrant Legumain inhibitor. Bioorg. Med. Chem. Lett. 25, 5642-5645.

Ness, K.A., Eddie, S.L., Burton, S., Harrison, T., Mullan, P., and Williams, R. (2016). Flat SAR of P3-methylsulphonamide based small molecule legumain inhibitors. Bioorg. Med. Chem. Lett. 26, 413-416.

Niestroj, A.J., Feussner, K., Heiser, U., Dando, P.M., Barrett, A., Gerhartz, B., and Demuth, H.U. (2002). Inhibition of mammalian legumain by Michael acceptors and AzaAsn-halomethylketones. Biol. Chem. 383, 1205-1214.

Olson, O.C. and Joyce, J.A. (2013). Microenvironment-mediated resistance to anticancer therapies. Cell Res. 23, 179-181.

Ovat, A., Muindi, F., Fagan, C., Brouner, M., Hansell, E., Dvorak, J., Sojka, D., Kopacek, P., McKerrow, J.H., Caffrey, C.R., et al. (2009). Aza-peptidyl Michael acceptor and epoxide inhibitors - potent and selective inhibitors of Schistosoma mansoni and Ixodes ricinus legumains (asparaginyl endopeptidases). J. Med. Chem. 52, 7192-7210.

Paulick, M.G. and Bogyo, M. (2008). Application of activity-based probes to the study of enzymes involved in cancer progression. Curr. Opin. Genet. Dev. 18, 97-106.

Peters, C. and Brown, S. (2015). Antibody-drug conjugates as novel anti-cancer chemotherapeutics. Biosci. Rep. 35.

Pistritto, G., Trisciuoglio, D., Ceci, C., Garufi, A., and D’Orazi, G. (2016). Apoptosis as anticancer mechanism: function and dysfunction of its modulators and targeted therapeutic strategies. Aging (Albany) 8, 603-619.

Poreba, M., Strozyk, A., Salvesen, G.S., and Drag, M. (2013). Caspase substrates and inhibitors. Cold Spring Harb. Perspect. Biol. 5, a008680.

Poreba, M., Szalek, A., Kasperkiewicz, P., Rut, W., Salvesen, G.S., and Drag, M. (2015). Small molecule active site directed tools for studying human caspases. Chem. Rev. 115, 12546-12629.

Poreba, M., Solberg, R., Rut, W., Lunde, N.N., Kasperkiewicz, P., Snipas, S.J., Mihelic, M., Turk, D., Turk, B., Salvesen, G.S., et al. 
(2016). Counter selection substrate library strategy for developing specific protease substrates and probes. Cell Chem. Biol. 23, 1023-1035.

Poreba, M., Salvesen, G.S., and Drag, M. (2017a). Synthesis of a HyCoSuL peptide substrate library to dissect protease substrate specificity. Nat. Protoc. 12, 2189-2214.

Poreba, M., Szalek, A., Rut, W., Kasperkiewicz, P., RutkowskaWlodarczyk, I., Snipas, S.J., Itoh, Y., Turk, D., Turk, B., Overall, C.M., et al. (2017b). Highly sensitive and adaptable fluorescence-quenched pair discloses the substrate specificity profiles in diverse protease families. Sci. Rep. 7, 43135.

Rawlings, N.D., Barrett, A.J., Thomas, P.D., Huang, X., Bateman, A., and Finn, R.D. (2018). The MEROPS database of proteolytic enzymes, their substrates and inhibitors in 2017 and a comparison with peptidases in the PANTHER database. Nucleic Acids Res. 46, D624-D632.

Rooseboom, M., Commandeur, J.N., and Vermeulen, N.P. (2004). Enzyme-catalyzed activation of anticancer prodrugs. Pharmacol. Rev. 56, 53-102.

Rotari, V.I., Dando, P.M., and Barrett, A.J. (2001). Legumain forms from plants and animals differ in their specificity. Biol. Chem. 382, 953-959.

Rozman-Pungercar, J., Kopitar-Jerala, N., Bogyo, M., Turk, D., Vasiljeva, O., Stefe, I., Vandenabeele, P., Bromme, D., Puizdar, V., Fonovic, M., et al. (2003). Inhibition of papain-like cysteine proteases and legumain by caspase-specific inhibitors: when reaction mechanism is more important than specificity. Cell Death Differ. 10, 881-888.

Ruppel, A., Shi, Y.E., Wei, D.X., and Diesfeld, H.J. (1987). Sera of Schistosoma japonicum-infected patients cross-react with diagnostic 31/32 kD proteins of S. mansoni. Clin. Exp. Immunol. 69, 291-298.

Saghatelian, A., Jessani, N., Joseph, A., Humphrey, M., and Cravatt, B.F. (2004). Activity-based probes for the proteomic profiling of metalloproteases. Proc. Natl. Acad. Sci. U.S.A. 101, 10000-10005.

Sanman, L.E. and Bogyo, M. (2014). Activity-based profiling of proteases. Annu. Rev. Biochem. 83, 249-273.

Schomburg, I., Chang, A., Ebeling, C., Gremse, M., Heldt, C., Huhn, G., and Schomburg, D. (2004). BRENDA, the enzyme database: updates and major new developments. Nucleic Acids Res. 32, D431-D433.

Schwarz, G., Brandenburg, J., Reich, M., Burster, T., Driessen, C., and Kalbacher, H. (2002). Characterization of legumain. Biol. Chem. 383, 1813-1816.

Sexton, K.B., Witte, M.D., Blum, G., and Bogyo, M. (2007). Design of cell-permeable, fluorescent activity-based probes for the lysosomal cysteine protease asparaginyl endopeptidase (AEP)/ legumain. Bioorg. Med. Chem. Lett. 17, 649-653.

Sheldon, P.S., Keen, J.N., and Bowles, D.J. (1996). Post-translational peptide bond formation during concanavalin A processing in vitro. Biochem. J. 320, 865-870.

Shree, T., Olson, O.C., Elie, B.T., Kester, J.C., Garfall, A.L., Simpson, K., Bell-McGuinn, K.M., Zabor, E.C., Brogi, E., and Joyce, J.A. (2011). Macrophages and cathepsin proteases blunt chemotherapeutic response in breast cancer. Genes Dev. 25, 2465-2479.

Singh, Y., Palombo, M., and Sinko, P.J. (2008). Recent trends in targeted anticancer prodrug and conjugate design. Curr. Med. Chem. 15, 1802-1826.
Smith, R., Johansen, H.T., Nilsen, H., Haugen, M.H., Pettersen, S.J., Maelandsmo, G.M., Abrahamson, M., and Solberg, R. (2012). Intra- and extracellular regulation of activity and processing of legumain by cystatin E/M. Biochimie 94, 2590-2599.

Smith, R.L., Astrand, O.A., Nguyen, L.M., Elvestrand, T., Hagelin, G., Solberg, R., Johansen, H.T., and Rongved, P. (2014). Synthesis of a novel legumain-cleavable colchicine prodrug with cellspecific toxicity. Bioorg. Med. Chem. 22, 3309-3315.

Stern, L., Perry, R., Ofek, P., Many, A., Shabat, D., and Satchi-Fainaro, R. (2009). A novel antitumor prodrug platform designed to be cleaved by the endoprotease legumain. Bioconj. Chem. 20, 500-510.

Tanaka, T., Inazawa, J., and Nakamura, Y. (1996). Molecular cloning of a human cDNA encoding putative cysteine protease (PRSC1) and its chromosome assignment to $14 q 32.1$. Cytogenet. Cell Genet. 74, 120-123.

Thornberry, N.A., Rano, T.A., Peterson, E.P., Rasper, D.M., Timkey, T., Garcia-Calvo, M., Houtzager, V.M., Nordstrom, P.A., Roy, S., Vaillancourt, J.P., et al. (1997). A combinatorial approach defines specificities of members of the caspase family and granzyme B. Functional relationships established for key mediators of apoptosis. J. Biol. Chem. 272, 17907-17911.

Vidmar, R., Vizovisek, M., Turk, D., Turk, B., and Fonovic, M. (2017). Protease cleavage site fingerprinting by label-free in-gel degradomics reveals $\mathrm{pH}$-dependent specificity switch of legumain. EMBO J. 36, 2455-2465.

Wallin, H., Apelqvist, J., Andersson, F., Ekstrom, U., and Abrahamson, M. (2017). Low-level internalization of cystatin E/M affects legumain activity and migration of melanoma cells. J. Biol. Chem. 292, 14413-14424.

Wang, Y., Jiang, Y., Zhang, M., Tan, J., Liang, J., Wang, H., Li, Y., He, H., Yang, V.C., and Huang, Y. (2014). Protease-activatable hybrid nanoprobe for tumor imaging. Adv. Funct. Mater. 24, 5443-5453.

Winter, A., Schmid, R., and Bayliss, R. (2015). Structural insights into separase architecture and substrate recognition through computational modelling of caspase-like and death domains. PLoS Comput. Biol. 11, e1004548.

Wu, W., Luo, Y., Sun, C., Liu, Y., Kuo, P., Varga, J., Xiang, R., Reisfeld, R., Janda, K.D., Edgington, T.S., et al. (2006). Targeting cellimpermeable prodrug activation to tumor microenvironment eradicates multiple drug-resistant neoplasms. Cancer Res. 66, 970-980.

Yuan, Y., Ge, S., Sun, H., Dong, X., Zhao, H., An, L., Zhang, J., Wang, J., Hu, B., and Liang, G. (2015). Intracellular self-assembly and disassembly of ${ }^{19} \mathrm{~F}$ nanoparticles confer respective "off" and "on" ${ }^{19} \mathrm{~F}$ NMR/MRI signals for legumain activity detection in zebrafish. ACS Nano 9, 5117-5124.

Zauner, F.B., Dall, E., Regl, C., Grassi, L., Huber, C.G., Cabrele, C., and Brandstetter, H. (2018). Crystal structure of plant legumain reveals a unique two-chain state with ph-dependent activity regulation. Plant Cell 30, 686-699.

Zeeuwen, P.L., van Vlijmen-Willems, I.M., Olthuis, D., Johansen, H.T., Hitomi, K., Hara-Nishimura, I., Powers, J.C., James, K.E., op den Camp, H.J., Lemmens, R., et al. (2004). Evidence that unrestricted legumain activity is involved in disturbed epidermal cornification in cystatin M/E deficient mice. Hum. Mol. Genet. 13, 1069-1079.

Zhang, Z., Song, M., Liu, X., Kang, S.S., Kwon, I.S., Duong, D.M., Seyfried, N.T., Hu, W.T., Liu, Z., Wang, J.Z., et al. (2014). 
Cleavage of tau by asparagine endopeptidase mediates the neurofibrillary pathology in Alzheimer's disease. Nat. Med. 20, 1254-1262.

Zhao, L., Hua, T., Crowley, C., Ru, H., Ni, X., Shaw, N., Jiao, L., Ding, W., Qu, L., Hung, L.W., et al. (2014). Structural analysis of asparaginyl endopeptidase reveals the activation mechanism and a reversible intermediate maturation stage. Cell Res. 24, 344-358.
Zhao, Y., Hai, Z., Wang, H., Su, L., and Liang, G. (2018). Legumainspecific near-infrared fluorescence "turn on" for tumor-targeted imaging. Anal. Chem. 90, 8732-8735.

Zhen, Y., Chunlei, G., Wenzhi, S., Shuangtao, Z., Na, L., Rongrong, W., Xiaohe, L., Haiying, N., Dehong, L., Shan, J., et al. (2015). Clinicopathologic significance of legumain overexpression in cancer: a systematic review and meta-analysis. Sci. Rep. 5, 16599. 